\title{
Advanced numerical method for generation of three- dimensional particles and its application in microstructure-based simulation of fatigue behavior
}

\author{
M. Milesi ${ }^{\mathrm{a},}{ }^{*}$, P.O. Bouchard ${ }^{\mathrm{a}}$, P.H. Cornuault ${ }^{\mathrm{b}}$, Y. Chastel $^{\mathrm{a}}$, M. Bernacki ${ }^{\mathrm{a}}$, E. \\ Hachem $^{\mathrm{a}}$ \\ ${ }^{a}$ MINES Paristech, Center for Materials Forming (CEMEF) UMR CNRS 7635 B.P. 20706904 \\ Sophia-Antipolis Cedex - FRANCE \\ ${ }^{b}$ MATEIS - Insa Lyon, 25, Avenue Jean Capelle 69621 Villeurbanne cedex - France
}

\begin{abstract}
The topology of representative elementary volumes (REV) generated to model materials microstructure is getting more and more complex. This paper presents advanced mesh generation methods used to improve the description of 3D microstructural particles. The goal is to adapt easily the shape of the elements at the interface between the isotropic matrix and embedded inclusions. Two methods are described in this work to generate inclusions: an analytical method based on statistical experimental data and a reconstruction approach, based on tomographic imaging. Sensitivity analyses on meshing parameters are performed to obtain efficient data in order to reconstruct the most representative volume and to perform subsequent accurate numerical computations. As an example of calculations, fatigue tests are chosen to validate the proposed approach.
\end{abstract}

\section{Keyword}

Anisotropic remeshing - 3D particles - X-ray tomography

\section{INTRODUCTION}

Numerical tools are getting more and more accurate to generate realistic microstructural meshes, with complex and realistic shapes of particles. First meshing inclusion generators were applied to represent aggregates in concrete materials. These pioneering studies have given rise to the classification of many empirical and analytical shapes determined from two-dimensional observations [1]. Numerical representation of particles began with simplified analytical forms like spherical inclusions. Different methods were developed to counterfeit numerical difficulties like "wall-effects", statistical representation of inclusions as well as numerical interface with finite elements. Previous methods considered positions and shapes of inclusions randomly as described in the work of Comby [2] with a rough representation of the interfaces between matrix and inclusions, depending on the initial mesh refinement. However, an adapted meshing procedure with concentric elements defining correctly the interface was proposed

*Corresponding author

Email addresses: marc.milesi@mines-paristech.fr 
by Sadouki [3] in 2D and generalized by Roelfstra [4] in 3D. To improve realistic microstructure representations, Pierard and Segurado $[5,6]$ developed a periodic microstructure with a random generation (random adsorption algorithm) in order to solicit an elementary volume with periodic strains. Shapes were also improved by considering more complex forms like ellipsoid [7] or angular forms [8]. To generate a more realistic REV, statistical methods were developed. An innovative method, proposed by Yang et al. [9], takes into account statistical forms and inclusion positions (see figure 1). The first method described concerns the "cellular segregation" consisting in positioning all inclusions by considering forbidden spaces like spherical voids. The second method is the "localized cluster" approach, retained in a previous work by Milesi and coworkers [10,11]. This method consists in restraining, from a statistic mean and a standard deviation, the width, the length and the height of the area where inclusions are generated. This method is very powerful to generate clusters of inclusions if all morphological characteristics are known.

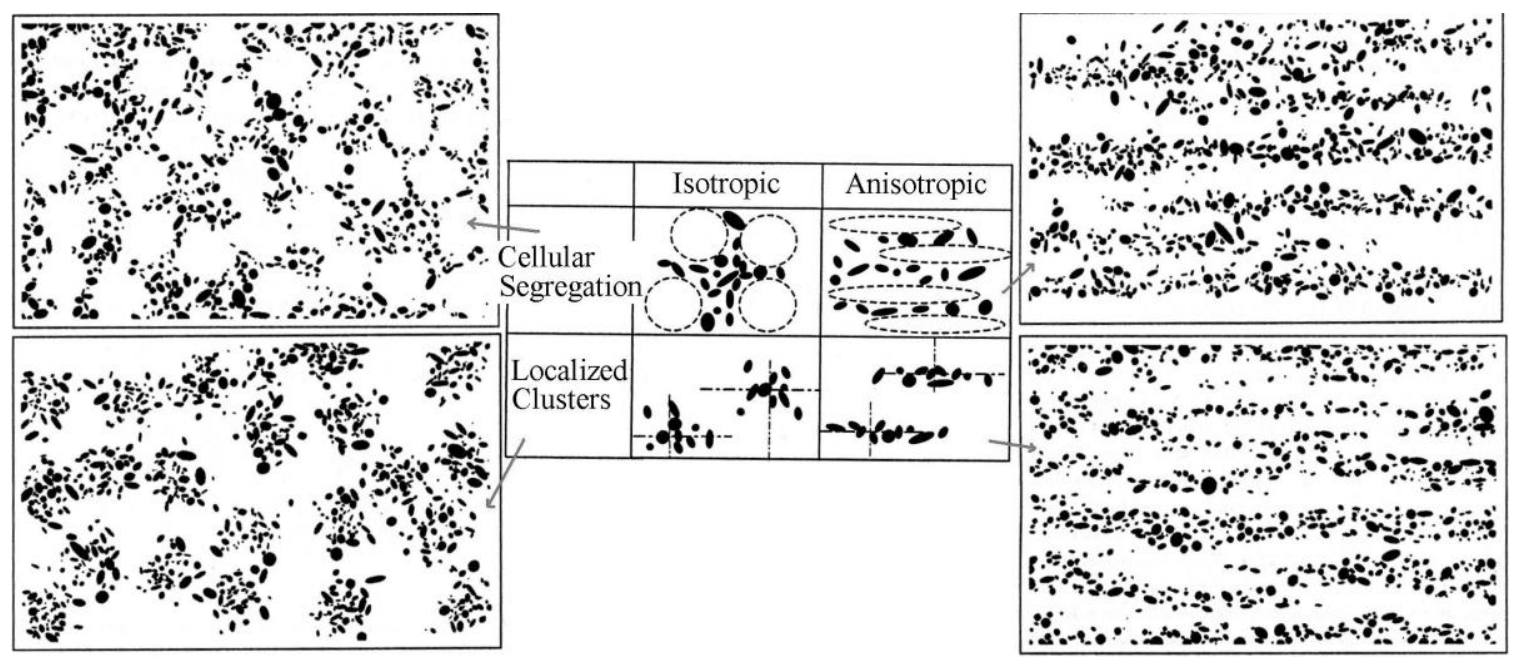

Figure 1: Statistical selection of inclusion positions by means of cellular segregation method and localized cluster method.

Methods based on imaging generations are also used to reconstruct microstructures. Chawla et al. $[12,13]$ proposed a method based on mechanical polishing and 2D imaging reconstruction. Tomographic reconstructions are also widely used nowadays, especially for medical imaging. This technique offers non destructive analyses to probe the interior of an opaque material without affecting the structure. Stiénon and coworkers [14] used micro-tomography to perform simulations on one $\mathrm{CaO}$ inclusion in order to assess the sensitivity of particles shapes and interfaces friction laws. Laschet et al. [15] also work on metallic foams reconstructed from X-ray microtomography to perform a multiscale approach. However, in both cases, the meshing method applied was not precisely described. 
Recall that the study of one single inclusion is not sufficient to understand all occurring interactions. In [10, 11], the authors have shown the significative influence of MnS inclusions clusters topology on high cycle fatigue computations at the microscale. But so far, generated elementary forms were only composed by simple ellipsoidal inclusions. It requires at least 12,000,000 finite element meshes to improve the stress field around inclusions. Consequently, for complex shapes, the remeshing method used in this previous study was not efficient enough to capture all irregularities for real inclusions obtained by tomographic reconstruction.

In this work, we propose then to improve the approach developed previously by Milesi and coworkers [10, 11] by considering numerical tomographic reconstruction of MnS inclusions clusters. The goal is to simulate different configurations of microstructural REVs to predict critical area in fatigue computations. Additionally, a new advanced adaptive method is presented and used to optimize the generated mesh of extremely complex REVs in order to obtain stable results with the minimum number of elements.

The outline of the paper is as follows: first, we present a brief review on the mechanical problem and the anisotropic remeshing algorithm. In Section 3, we present two methods to reconstruct a 3D microstructure from statistical ellipsoidal inclusions and from tomographic imaging. The novel approach to generate and to obtain high quality meshes is described. In Section 4, the meshing parameters such as number of elements and the security distance are optimized based on a sensitivity analysis from a large number of numerical tests. Finally, conclusions and perspectives are outlined.

\section{MESH OPTIMIZATION AND MECHANICAL PROBLEM}

Simulations on REVs are directly governed by boundary conditions, volume fraction of inclusion and size of the REV. Sensitivity analyses of these different parameters on the determination of stress-strain curves have been studied by Gitman et al. [16] and gave an accurate overview of the difficulty to validate the size of a REV. Milesi et al. [10,11] have worked on complex geometries with cluster inclusions using a gradient remeshing method. The main hypothesis of this study is to consider a cluster of inclusions embedded in an isotropic steel matrix (verified by electron backscattered diffraction analyses). This method is based on the resolution of a single set of equations for the whole computational domain and the treatment of the different subdomains as a single geometrical domain, but with variable material properties. To separate each constituent from the matrix, the so-called level set method is applied [17] from an initial extremely refined mesh in order to associate the elements of the mesh to the subdomains composed by inclusions. In practice, a signed distance function is used to localize implicitly the interfaces and to associate different mechanical properties by means of mixing laws. Remeshing is an incremental method which, from an initial mesh, gives more and more refined elements near the interface or, on the 
contrary, removes targeted mesh elements far from interfaces. This interface can be directly controlled in terms of number of elements and thickness. In our context, the interface is static. Let $\Omega_{M a t}, \Omega_{I n c}$ and $\Gamma_{i}$ be respectively the matrix domain, the inclusion domain and the interface. They verify:

$$
\Omega_{M a t} \cup \Omega_{I n c}=\Omega \text { and } \Omega_{M a t} \cap \Omega_{I n c}=\Gamma_{i} .
$$

For each node (x representing the position) of the computational domain $\Omega$, the level set function $\alpha$, which is the signed distance from the interface, is described by the following conditions:

$$
\alpha(\mathrm{x})=\left\{\begin{array}{l}
>0 \text { if } \mathrm{x} \in \Omega_{\mathrm{Mat}} \\
=0 \text { if } \mathrm{x} \in \Gamma_{\mathrm{i}} \\
<0 \text { if } \mathrm{x} \in \Omega_{\mathrm{Inc}}
\end{array}\right.
$$

$\alpha$ is calculated and associated to each constituent of the material. If the number of constituents is $p$, then $p \times \alpha_{i}$ can be calculated (for $i=1 \ldots p$ ). Then $\alpha$ becomes:

$$
\alpha=\max _{i=1 \ldots p} \alpha_{i}
$$

The mechanical properties in the domain are then smoothed and calculated as a function of $\alpha$. For instance, the mixed hardening coefficient is calculated using a linear interpolation between the values of the hardening coefficient $n_{\text {mat }}$ in the matrix and $n_{i n c}$ in the inclusion:

$$
n=n_{m a t} H(\alpha)+n_{\text {inc }}(1-H(\alpha)),
$$

where $H$ is a smoothed Heaviside function given by:

$$
H(\alpha)= \begin{cases}1 & \text { if } \alpha>\varepsilon \\ \frac{1}{2}\left(1+\frac{\alpha}{\varepsilon}\right) & \text { if }|\alpha| \leq \varepsilon, \\ 0 & \text { if } \alpha<-\varepsilon\end{cases}
$$

where $\varepsilon$ is a small parameter such that $\varepsilon=\mathrm{O}\left(h_{i}\right)$, known as the interface thickness, and $h_{i}$ is the mesh size in the normal direction to the interface. In the vicinity of the interface, it can be computed using the following expression:

$$
h_{i}=\max _{j, l \in \mathrm{K}} \nabla \alpha \cdot \mathrm{x}^{\mathrm{j} 1}
$$


where $\mathrm{x}^{\mathrm{j} 1}=\mathrm{x}^{1}-\mathrm{x}^{\mathrm{j}}$ and $\mathrm{K}$ is the mesh element. Further details about the algorithm used to compute the distance are available in [18, 19, 20, 21]. In order to circumvent the high level of stress gradient due to different mechanical properties, the level-set process is coupled to an anisotropic mesh adaptation. The idea of this method is to pre-adapt in a fast way the mesh at the interface. The mesh becomes locally refined which enables to sharply define the interface and to save a great number of elements compared to standard isotropic refinement. This anisotropic adaptation is performed by constructing a metric map that allows the mesh size with different values in different direction. Metric is a symmetric defined positive tensor representing a local base that modify the distance computation, such that:

$$
\|x\|_{M}=\sqrt{{ }^{t} x \cdot M \cdot x}, \quad\langle x, y\rangle_{M}={ }^{t} x \cdot M \cdot y .
$$

The metric $\mathrm{M}$ can be regarded as a tensor whose eigenvalues are related to the size elements, and whose eigenvectors define the directions for which these sizes are applied. For instance, using the identity tensor, one recovers the usual distances and directions of the Euclidean space. In our case the direction of mesh refinement is given by the unit normal to the interface which corresponds to the gradient of the level set function: $\mathrm{x}=\nabla \alpha /\|\nabla \alpha\|$. A default mesh size, or background mesh size, $h_{d}$ is imposed far from the interface and it is reduced as the interface comes closer. An interesting choice for the mesh size evolution is the following:

$$
h= \begin{cases}h_{\mathrm{d}} & \text { if }|\alpha(\mathrm{x})|>\mathrm{e} / 2 \\ \frac{2 h_{\mathrm{d}}-1}{\mathrm{qe}}-\alpha(\mathrm{x}) \mid+\frac{h_{\mathrm{d}}}{\mathrm{q}} & \text { if }|\alpha(\mathrm{x})| \leq \mathrm{e} / 2\end{cases}
$$

Eventually, at the interface, the mesh size is reduced by a factor q with respect to the default value $h_{d}$. Then this size increases until equalling $h_{d}$ for a distance that corresponds to the half of a given thickness e. The unit normal to the interface $\mathrm{x}$ and the mesh size $h$ defined above, lead to the following metric:

$$
\mathrm{M}=C \varangle \otimes \mathrm{x} \ni \frac{1}{h_{d}} \mathrm{I} \quad \text { with } \quad \mathrm{C}= \begin{cases}0 & \text { if }|\alpha(\mathrm{x})| \geq \mathrm{e} / 2 \\ \frac{1}{\mathrm{~h}^{2}}-\frac{1}{\mathrm{~h}_{\mathrm{d}}^{2}} & \text { if }|\alpha(\mathrm{x})|<\mathrm{e} / 2\end{cases}
$$

where $I$ is the identity tensor. This metric returns to isotropic far from the interface (with a mesh size equals to $h_{d}$ for all directions) and to anisotropic near the interface (with a mesh size equals to $h_{i}$ in the direction of $\mathrm{x}$ and equals to $h_{d}$ in the others). More details can be found in [22]. In practice, the mesh is generated in several steps using, through the CimLib library, the MTC mesher and remesher developed by Coupez [23]. This method was applied successfully 
to construct a $12,000,000$ elements mesh. The minimal size of the mesh was fixed to $0.077 \mu \mathrm{m}$ (the REV size was $100 \times 100 \times 100 \mu \mathrm{m}^{3}$ ) and was corresponding to the interface thickness. Figure 2 shows the mesh around an inclusion at two different stages of the incremental remeshing procedure. It can be noticed that after 16 increments, the mesh is more precise and efficient around the inclusion than at the $6^{\text {th }}$ meshing increment.

To perform simulations, the geometry is defined and mechanical properties are assigned based on the signed distance function. The heterogeneous REV can now be dealt with a single domain whose effective properties are defined using mainly linear interpolations between their coefficients as previously defined in expression (4). The smoothed Heaviside function defined in (5) enables to assign the actual properties on each side of the interface. The global material properties such as the hardening coefficient $\mathrm{n}$, the consistency $\mathrm{K}$ and the Young modulus $\mathrm{E}$ are defined by the following laws:

$$
\begin{aligned}
& \mathrm{n}=\mathrm{n}_{\text {mat }} H(\alpha)+\mathrm{n}_{\text {inc }}(1-H(\alpha)) \\
& \mathrm{K}=\mathrm{K}_{\text {mat }} H(\alpha)+\mathrm{K}_{\text {inc }}(1-H(\alpha)) \\
& \mathrm{E}=\mathrm{E}_{\text {mat }} H(\alpha)+\sum_{i=1}^{\text {nbinclusions }} \mathrm{E}_{\text {inc }}^{\mathrm{i}}\left(1-H\left(\alpha_{i}\right)\right) \text { with } \sum_{i=1}^{\text {nbinclusions }} H\left(\alpha_{i}\right)=H(\alpha)
\end{aligned}
$$
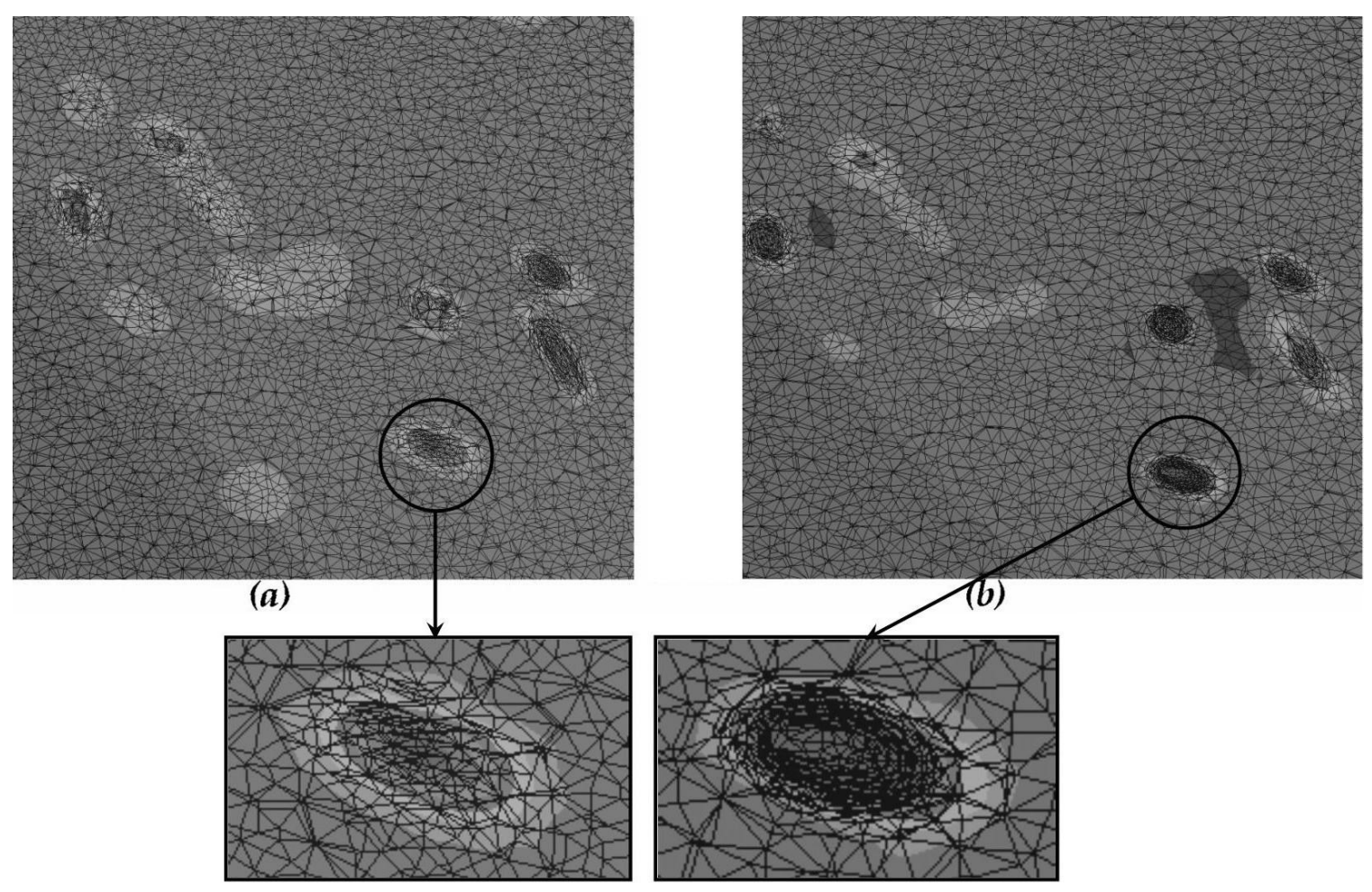

Figure 2: Incremental remeshing method: a) mesh after 6 remeshing increments and b) mesh obtained after 16 remeshing increments. 
All parameters related to the material of this study (a METASCO MC steel) are given in table 1. For the Young modulus, results have been determined by nanoindentation analyses [24]. The best statistical law determined to fit the inclusions behavior seemed to be a gaussian law [10] although for the matrix, the lognormal law seemed to match better the realistic behavior. However, given the isotropic assumption of the matrix and the small value of the standard deviation, the Young modulus of the matrix was fixed to the mean value. Inclusions behavior is assumed to be purely elastic whereas the matrix mechanical behavior is defined by an elastic-plastic power law.

\begin{tabular}{lrr}
\hline Properties & Steel matrix & Inclusions \\
\hline Hardening coefficient $\mathrm{n}$ & 0.15 & 1 \\
Consistency [MPa] & 1695 & - \\
Young modulus E [GPa] & $218.7 \pm 10.8$ & $118.8 \pm 40.5$ \\
\hline
\end{tabular}

Table 1: Properties of the considered material.

For the numerical resolution, a non-linear solver is used with a Newton-Raphson algorithm developed in the CimLib library [25]. This solver is based on a mixed finite element velocity-pressure formulation and uses the so-called mini-element $(\mathrm{P} 1+/ \mathrm{P} 1)$. The mini-element is a linear isoparametric tetrahedron (triangle for $2 \mathrm{D}$ problems) where a bubble function is added at its center to ensure stability. The numerical problem is non-linear due to the elastic-plastic law given by:

$$
\bar{\sigma}=K \sqrt{3}\left(\overline{\varepsilon_{0}}\right)
$$

where $(\bar{\sigma}, \bar{\varepsilon})$ corresponds respectively to the equivalent stress and equivalent strain, $\overline{\varepsilon_{0}}$ is the initial strain equals to $0, \mathrm{~K}$ is the consistency and $\mathrm{n}$, the hardening coefficient [26]. Symmetrical boundary conditions have been employed for stresses on two opposite faces and two other faces of the REV were also submitted to symmetrical conditions noted by:

$$
\begin{aligned}
& \underline{\underline{\sigma}} \cdot \underline{\mathrm{n}}=\mathrm{constant}, \\
& \underline{\mathrm{v}} \cdot \underline{\mathrm{n}}=0
\end{aligned}
$$

where $\underline{\mathrm{v}}$ is the velocity and $\underline{\mathrm{n}}$, the surface normal. Using symmetrical boundary conditions is a means to counterfeit the non periodicity of the REV due to the non homogeneous shape of inclusions. It is not a poly-crystalline matrix but only homogeneous one. Periodic boundary conditions are not needed in this case. Moreover, inclusions are imbedded within the REV with a security distance from 
borders which prevent from the numerical "wall-effect". No inclusion emerges towards the surface.

In order to compare results obtained for different mesh generation, a fatigue criterion was chosen to estimate the stress field around inclusions. Such criterion gives an indicator by taking into account the hydrostatic pressure, which can be benefic or detrimental for fatigue performance (it depends on the sign), as well as the shear stress. Milesi et al. [11] chose the Papadopoulos criterion [27] to quantify the impact of a high cycle fatigue loading on the material resistance. Two material coefficients are needed to validate this criterion. These coefficients depend on the material state and can be determined from cyclic pure tensilecompressive and torsion tests and can be written as:

$$
\frac{\mathrm{A}+\gamma \Sigma_{H, \max }}{\beta} \leq 1,
$$

which corresponds to a linear domain. $\Sigma_{H, \max }$ relates to the maximum hydrostatic stress over the loading cycle [27]. A is a shear stress parameter that depends on the class of material studied. $\gamma$ and $\beta$ can directly be calculated from the purely reversed tensile and torsion endurance limits of the material. More details can be found in [11, 27, 28, 29]. This normalized version of the Papadopoulos fatigue criterion will be used later in further details to compare the different methods of the 3D numerical generation. It can be noted that the fatigue resistance is related to the isotropic matrix resistance (not to inclusion damaging).

\section{NUMERICAL METHODS FOR MICROSTRUCTURAL PARTICLES}

These approaches are generally used to perform numerous simulations on various statistical based REVs, in order to obtain statistical results experimentally impossible to obtain due to the huge amount of configurations tested. The results obtained can be used for instance as input parameters of macroscopic fatigue criteria to account for anisotropy due to inclusions orientation. The first method described concerns the statistical generation of ellipsoidal inclusions already confronted and validated to experimental results in [11]. The second method presents the new procedure used for X-ray tomographic generation.

\subsection{STATISTICAL GENERATION OF INCLUSIONS}

Milesi et al. [10] applied such generations to perform high cycle fatigue computations on a forging component. The authors assumed the size of the representative elementary volume to a $100 \times 100 \times 100 \mu \mathrm{m}^{3}$ cube. Experimental analyses were performed in order to obtain accurate morphological and mechanical characteristics of the microstructure based on rigorous statistical 
data. Electron backscattered diffraction analyses indicated that the structure could be assessed as an isotropic granular structure. Only inclusions were assumed to be at the origin of the anisotropic behavior of the material. As demonstrated in [11], components high cycle fatigue properties were significantly reduced when clusters of elongated inclusions were perpendicular to the loading direction. To separate inclusions from the rest of all microstructural constituents, scanning electron microscope images were analysed by digital image processing techniques. All images were taken with a high definition and analysed by means of an imaging software. A rigorous protocol based on images thresholding in grey scale has been undertaken. To avoid any perturbations, an optimization procedure has been performed in the calculation of the different inclusion parameters. Only manganese sulfide MnS inclusions were selected in this study. Inclusions with a mean size lower than $1.4{\mu \mathrm{m}^{2}}^{2}$ were not taken into account in the generation. This threshold surface is used to avoid uncertainties in the image analysis procedure ; for instance, pollutions coming from OPS solution used for polishing. The authors have shown that the inclusions shapes were similar to ellipsoidal geometries, especially before forging (i.e. inclusions tortuosity was assumed negligible).

Numerical statistical selections were described by a Box-Müller method [30]. This method consists in generating a uniform normally distribution from two independent random variables. Random variables were carried out with the pseudo-random generator mersenne twister algorithm [31]. The Box-Müller method was also adapted to get a log-normal distribution [32]. Figure 3 gives an example of ellipsoidal generation of inclusions with the localized cluster method [9] and the remeshing method previously described. Some generated REVs were containing more than 300 inclusions gathered in a single cluster. Each parameter of the generation procedure can be customized. For example, Euler angles can be associated independently to each inclusion following a normal statistical law. Inclusions thickness, width and length can also be associated to a log-normal statistical law. Inclusions are generated by taking into account their number for each cluster, so that density (or volume fraction) can be easily controlled. A software dedicated to microstructural generation were specifically developed to generate such microstructures $[33,34,35]$. 


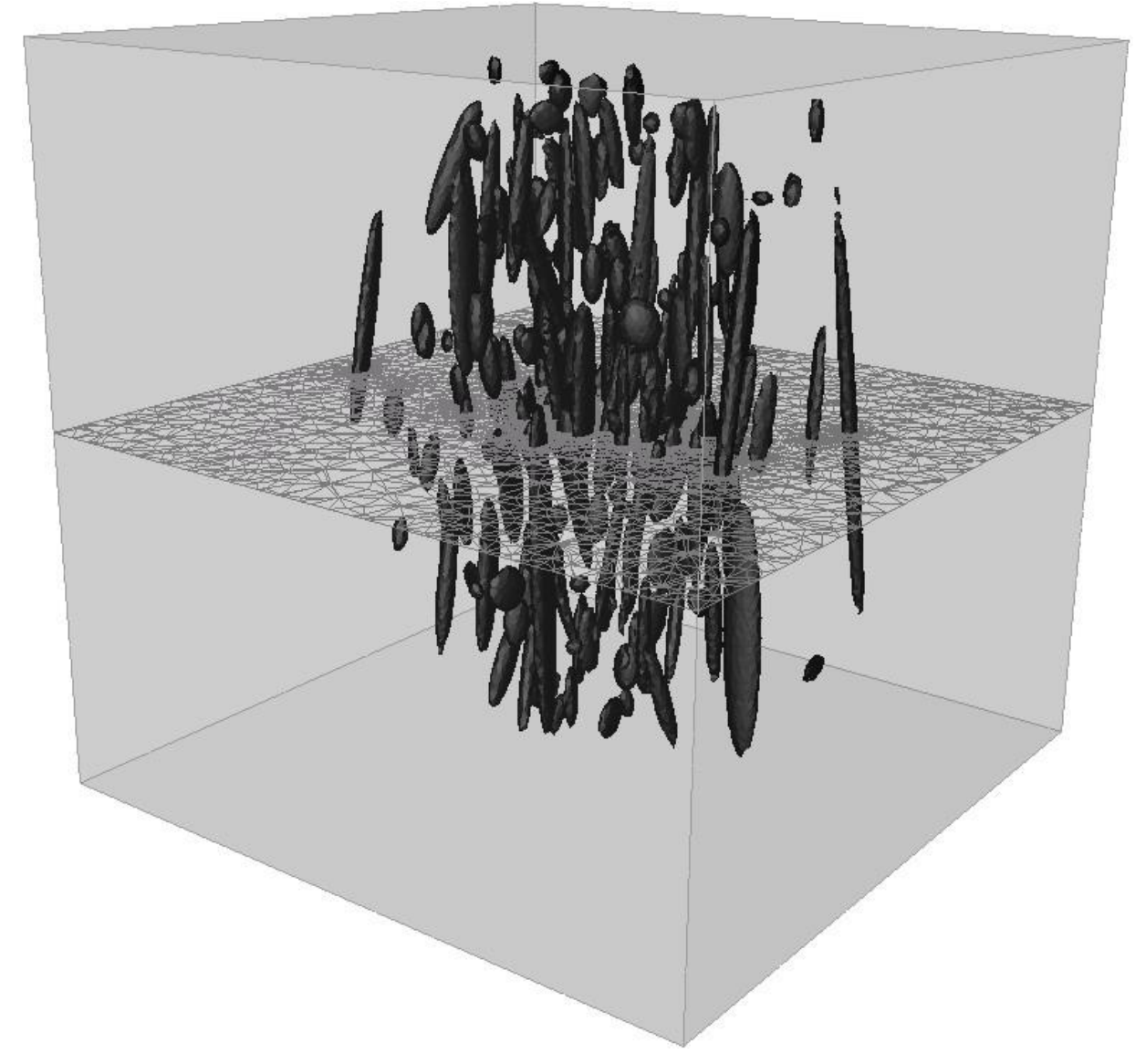

Figure 3: 220 ellipsoidal inclusions generated and remeshed from statistical data.

To counterfeit wall-effects, a security distance from the surface has been implemented. This security distance guaranty no stress localization near the surface. A security distance of $1 \mu \mathrm{m}$ has been employed to avoid stresses concentration near edges. This security distance has been proved to be sufficient for these ellipsoidal shapes.

\subsection{GENERATION BASED ON X-RAY TOMOGRAPHY ANALYSES\}}

In order to improve digital computations on a REV, tomographic reconstruction of the real material has been undergone. The idea is to account for the tortuosity of inclusions which cannot be well represented using ellipsoidal forms. Indeed, strains induced during the forming process can have a major influence on the shape of inclusions, especially the tortuosity $\tau$. Tortuosity can be defined by the apparent length $L_{a p p}$ of the inclusion over its real length $L_{\text {real }}$ if you stretched it until obtaining no folding. This idea can be summed up by the following simplified equation: 


$$
\tau=\frac{L_{a p p}}{L_{\text {real }}} .
$$

In the present work, tortuosity reaches an average of 0.7 after the forging process and sometimes far less (it depends on the studied area because deformation state is not homogeneous). The industrial component studied undergone seven stages of forging including crushing, cambering, coating and cambering operations. Each stage performs a high level of deformation which could be mechanically described by the kneading rate inflicted during all process. Due to this, inclusions undergone a high local strain which deforms them (or even damages them) in the direction of the microstructural flow. The resulting shape is represented in figure 4 illustrating a reconstruction by tomographic probe.

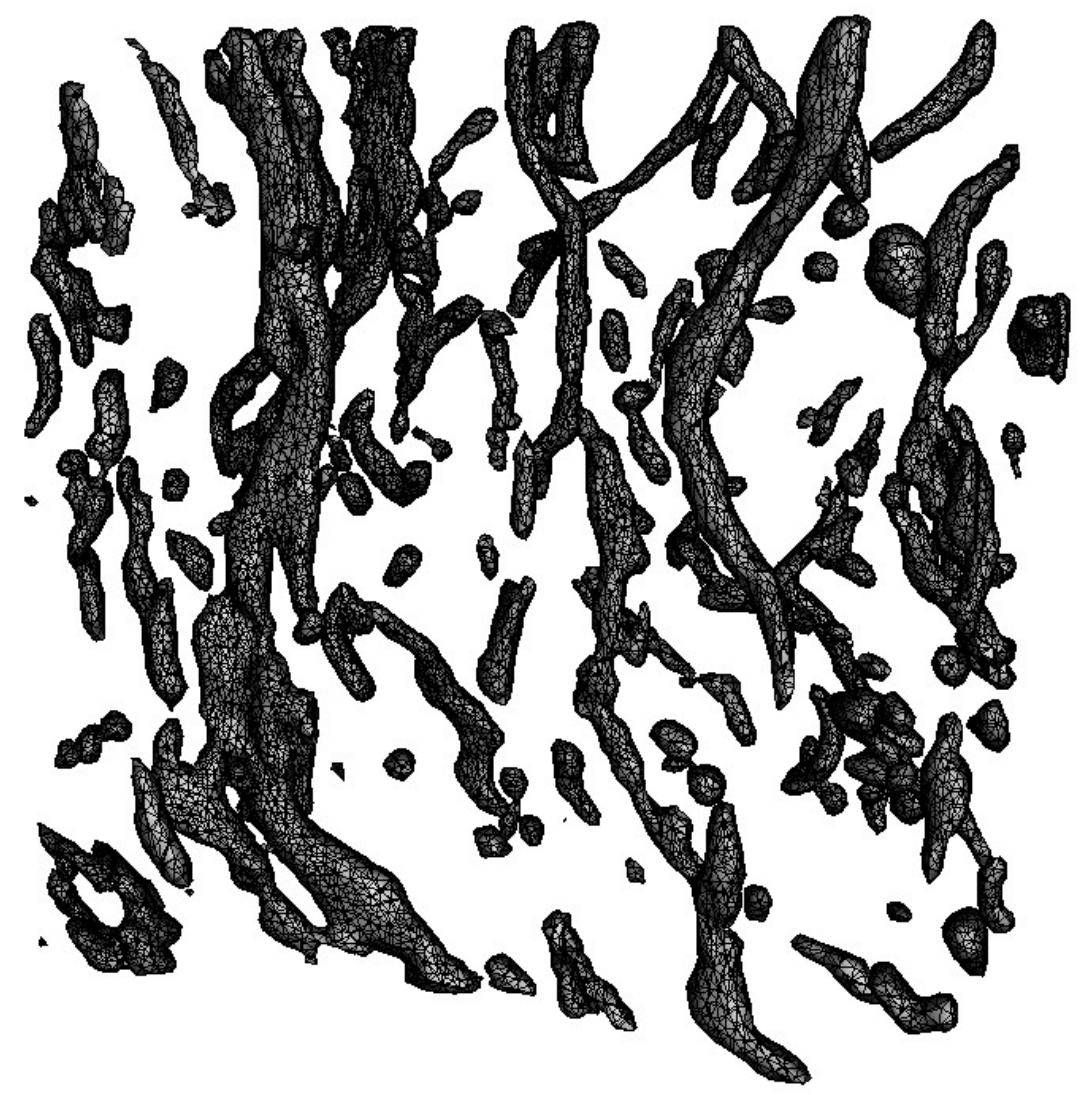

Figure 4: Tomographic reconstruction of inclusions with a high level of tortuosity.

X-ray tomography characterization was performed on both components before and after forging in order to study the influence of tortuosity. Because of the high mass attenuation coefficient of the investigated material, thin samples are needed. Consequently, two $500 \mu \mathrm{m} \times 500 \mu \mathrm{m} \times 20 \mathrm{~mm}$ plane-parallel sticks were extracted from each part (before and after forging) by means of electro-discharge machining. Attention was paid to extract samples far from the center and outer 
surfaces of the initial components in order to avoid inclusions clusters concentration variations observed in these zones. X-ray imaging of samples was performed in the ID19 line at the European Synchrotron Radiation Facility (ESRF). After the 2D projections acquisition, a classical filtered back projection algorithm developed at ESRF allows to reconstruct a 3D numerical image of the sample portion [36]. After 3D reconstruction, an initial stack of 2000 slices was obtained, each slice corresponding in a $2 \mathrm{D}$ reconstructed image of a stick crosssection which is parallel to its base. Figure 5 a) depicts an example of one slice extracted from a non-forged component where matrix appears in light-gray and inclusions in black. Every slice was separated from the following one by a $0.7 \mu \mathrm{m}$ distance.

Then, the four stacks obtained (two for each component) were equally treated using an imaging software. First, a $100 \times 100 \times 100 \mu \mathrm{m}^{3}$ volume was cropped into the whole stack. Deliberately, the volume location was arbitrarily chosen. Matrix was eliminated using a thresholder selecting only pixels defining inclusions. Then, slices were binarised. Finally, the inclusions having a volume smaller than 27 voxels (about $9 \mu^{3}$ ) have been excluded. Figure 5 b) shows representative results obtained after cropping, noise filtering, thresholding, binarisation and cleaning of the same stack slice than figure 5 a). Finally, the resulting stacks were imported in the Amira software where a continuity hypothesis between every slice was applied and an automatic surface mesh reconstruction was performed.

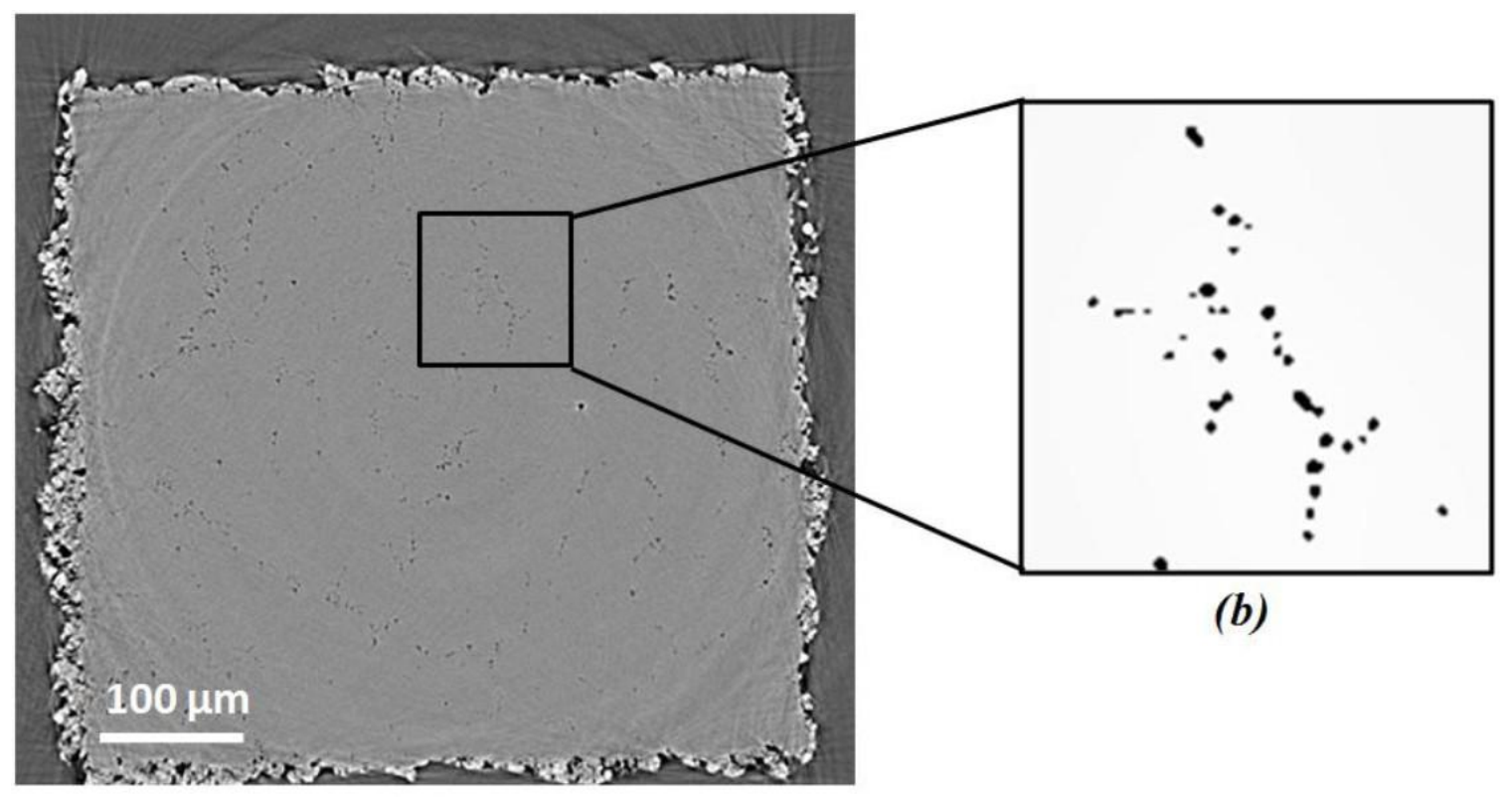

(a)

Figure 5: a) One slice extracted from a stack coming from the non-forged component after 3D reconstruction and $b$ ), slice after cropping, noise filtering, thresholding, binarisation and cleaning. Inclusions appear in black. 
To obtain the mesh shown in figure 4, the remeshing method described previously is not sufficient due to the complicated shapes of so many inclusions. Indeed, the main drawback of this approach is the necessity to begin the remeshing procedure with an initial mesh already extremely refined isotropically in order to associate mesh elements to each level set function. If the initial mesh does not contain sufficiently small size elements, the level set can not be built. Indeed, the smaller inclusion size is, the higher number of elements of the initial mesh is needed. This meshing technique is well suited to regular geometries like ellipsoidal shapes, but the method is less efficient with complex shapes. Another methodology is required. The mesh adaptation used is based on the determination of the second derivatives hessian matrix of the level set function (the so-called Hessian strategy [37, 38, 39, 40]). The goal of this approach is to achieve a mesh-adaptative strategy minimizing the directional error estimation in the mesh. By this means, it is possible to refine the mesh close to the microstructural particles, stretch and orient the elements in such a way that, along the adaptative process, not only accurate representation of the level set are obtained but also it tolerates keeping the number of unknowns affordably low. The Hessian strategy is used to obtain better directional information of the error. The level set scalar component is used to compute the Hessian. As shown in previous section, this directional information can be converted into a mesh metric field $M$ (equation 9) which prescribes the desired element size and orientation. However, it was applied as an 'adhoc' method without an error estimator or error control. Following the methodology presented in [38, 40,41], we first introduce an anisotropic bound of the error interpolation:

$$
\left\|e_{k}\right\|_{\infty} \leq c \max _{\mathrm{x} \in \mathrm{K}} \max _{e}\left(H_{u}(\mathrm{x}) \mid e_{-}^{-},\right.
$$

where $c$ is a constant that depends on the space dimension, $e$ denotes the edges of each element $\mathrm{K}$ and $\left|H_{u}(\mathrm{x})\right|$ is the absolute value of the Hessian matrix of the level set solution (i.e. a symmetric definite positive tensor). Note that the Hessian strategy involves the computation of the symmetric matrix of second derivatives and can be decomposed as $\left|H_{u}(\mathrm{x})\right|=R|\Lambda| R^{T}$, where $R$ is the eigenvectors matrix and $|\Lambda|=\operatorname{diag}\left(\lambda_{k}\right)$ is the diagonal matrix of eigenvalues. The directions associated with the eigenvectors $k$ are referred to principal directions and the eigenvalues $k$ are then equivalent to the second derivatives along the local principal directions. Since the Hessian strategy is based on the idea that a high magnitude of an eigenvalue implies a high error in the direction associated with the corresponding eigenvector, consequently a small element size would be desired in this direction along the inclusions interfaces. Then again, a low eigenvalue magnitude in a particular eigendirection would lead to a large element size along this direction far from the interfaces of the microstructural particles. In order to achieve such procedure and to obtain a suitable mesh resolution in 
different directions, we apply a uniform distribution of local errors in the principal directions leading to:

$$
\varepsilon=\operatorname{ch}_{k}^{2} \overline{\lambda_{k}},
$$

where $\varepsilon$ is the user specified tolerance for the error and $h_{k}$ is the desired size in the $k^{\text {th }}$ principal direction. So, the edges $e$ of the adapted mesh must ensure:

$$
\varepsilon=c(\mathrm{Me}
$$

The stated goal of the mesh adaptation algorithm is to yield a mesh with regular elements in the metric space where each edge $e$ must satisfy the following relation:

$$
e \cdot \overline{\mathrm{M}} e=1 .
$$

A mesh with all its edges satisfying the above relationship is commonly referred to a unit mesh. Finally, a mesh metric tensor $\overline{\mathrm{M}}$ is obtained at each node by calculating a scaled eigenspace of the recovered Hessian matrix as $\bar{M}=R|\bar{\Lambda}| R^{T}$, where $R$ is the eigenvector matrix and $|\bar{\Lambda}|=\frac{c}{\varepsilon} \Lambda$ is the diagonal matrix of scaled eigenvalues.

An extra control is added and truncation values $h_{\min }$ and $h_{\max }$ are specified to limit mesh sizes. One reason for truncating the element size, in terms of edge lengths, is to avoid singular metrics. For example, it is necessary to apply $h_{\max }$ in case of a null or quasi-null eigenvalue in the direction where the solution does not vary. The modified eigenvalues of the Hessian matrix become then:

$$
\bar{\lambda}_{k}=\min \left(\max \left(\frac{c}{\varepsilon}\left|\lambda_{k}\right|, \frac{1}{h_{\max }^{2}}\right), \frac{1}{h_{\min }^{2}}\right) .
$$

The surface mesh given by Amira software is not connex, meaning that the mesh file is an entire block constituted by separated particles. A numerical method was developed to separate each inclusion in order to obtain connex meshes for all inclusions with tetrahedron elements. Each inclusion has been separated to employ the same method of remeshing refinement based on the Hessian strategy. The volume fraction is obtained by summing all tetrahedron volumes composing each inclusion. For the five meshes referencing the part before forging (obtained by tomography), the mean value and the standard deviation of the volume fraction calculated equals respectively to $1.006 \%$ and $0.332 \%$. The mean value and the standard deviation of the volume fraction calculated from five 
tomographic meshes corresponding to the component after forging are respectively $0.944 \%$ and $0.328 \%$.

\section{MECHANICAL COMPUTATIONS AND SENSITIVITY ANALYSES}

To validate the Hessian strategy, two different configurations of REVs were generated. The first one (stemming from the non forging part) contains relatively straight inclusions whereas the second one (the forging part) is composed by extremely deformed inclusions due to the high level of deformation. Mesh, Young modulus, security distance from edges and number of mesh elements describe all parameters tested and improved to define an optimized strategy. The purpose is to validate this methodology in terms of numerical convergence (in space) and in terms of reduction of the number of elements compared to the previous method used in [11] with a comparable time of computation. The optimized strategy is based on numerical tests oriented towards fatigue computations as previously described.

The first simulation concerns the raw REV of the part before forging directly obtained from Amira software. Cyclic tension-compression simulations have been carried out on a $102 \times 102 \times 102 \mu \mathrm{m}^{3}$ cube and composed of 212 inclusions. It is important to note that inclusions were defined in a $100 \times 100 \times 100 \mu^{3}$ cube by means of tomographical reconstruction and were centered in the cube of $102 \times 102 \times 102{\mu \mathrm{m}^{3}}^{3}$. It means that the security distance equals to $1 \mu \mathrm{m}$. A representation of this REV is given into two views in figure 6 . It can be noticed that inclusion shapes are straight, consequence of the rolling process. The main deformation is oriented in the rolling direction and transverse deformations are negligible. In order to study the mesh sensitivity, fatigue loadings were applied every five increments of the remeshing hessian strategy with the estimation method. 70 increments were needed to obtain a 6,000,000 millions element volume. These meshes were obtained by means of parallel calculations on 16 processors of a 512 cores Opteron $2.4 \mathrm{GHz}$ linux cluster. The time of the remeshed method depends on the number of inclusions but it can be estimated to 72 hours for the most refined mesh. 


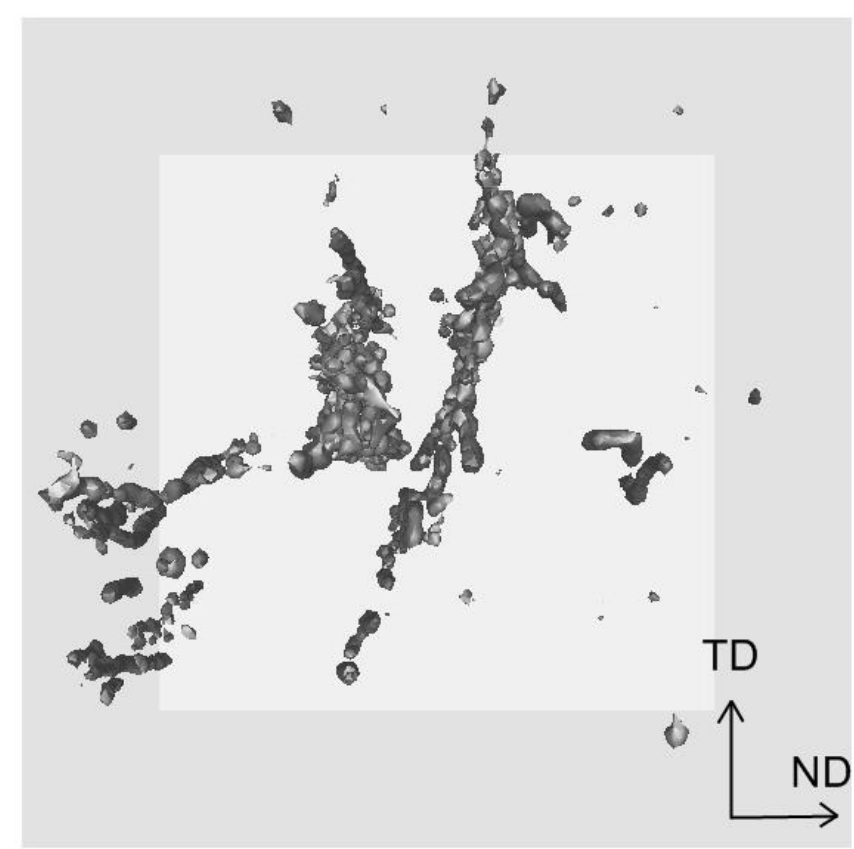

(a)

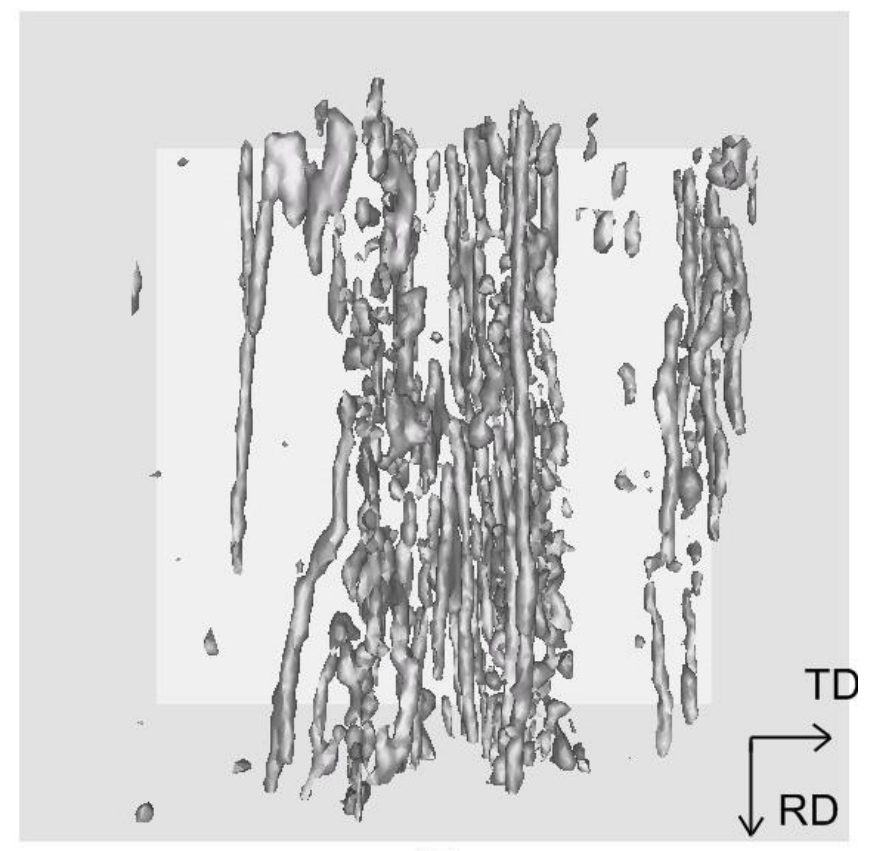

(b)

Figure 6: Numerical tomographic sample represented in a) the normal direction (ND) - transverse direction (TD) and in b) the rolling direction (RD) - transverse direction (TD).

Figure 7 gives the evolution of the most critical value of the Papadopoulos criterion [27] compared to the evolution of the number of mesh elements obtained at different steps of the incremental remeshing method. The remeshing method is incremental, meaning that the optimization loop refines more and more the mesh 
by considering the entire inclusion shapes (estimation method). This value rises to stabilize toward 5,500,000 elements and reaches an asymptote. The relative error is calculated from the final result.

However, the total volume referencing each inclusion tends to converge to the real volume fraction after several increments. Figure 8 displays the correlation between the number of elements and the volume fraction of inclusions during incremental remeshing. It can be noticed that after several increments, the volume fraction reaches exponentially the real volume fraction of inclusions preforms given initially. Papadopoulos critical value is also plotted with a different scale. The evolution is also exponentially and very well correlated with the previous curve. When the value of the volume fraction is very close to the real value, the prediction of the Papadopoulos criterion stops scattering and converges towards a constant value. The relation between these two parameters (volume fraction and Papadopoulos critical value) seems to be very well correlated. Each further REV was constructed by considering the convergent volume fraction as a limitation for the number of mesh elements.

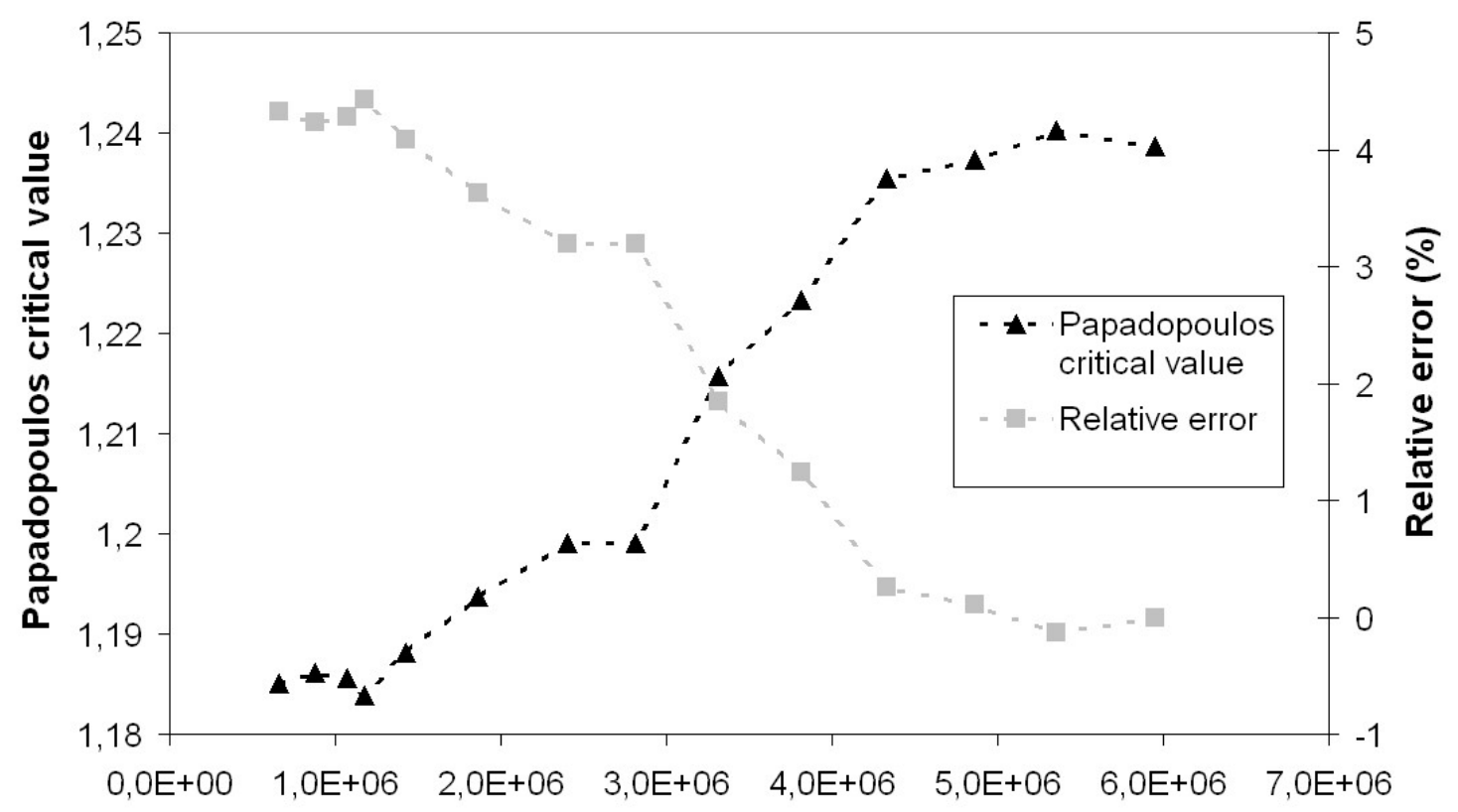

Number of mesh elements

Figure 7: Evolution of the Papadopoulos critical value depending on the remeshing method increments.

To gauge the impact of "wall effects", different security distances have been chosen. Results are given in figure 9. They reveal that for a value close to $5 \mu \mathrm{m}$, the Papadopoulos critical value tends to stabilize. This distance remains lower than the mean value of the inter-distance of cluster given by Milesi [10]. Then, the influence between each cluster is not disrupted by accounting for this security 
distance. By considering such a distance, the detrimental "wall-effects" are then dodged. It can be noted that several inclusions are localized close to the border of the volume. It can induce an increase of the stress localization for a non adapted security distance as represented in figure 10 for a security distance of 1 $\mu \mathrm{m}$. All fatigue simulations were parallelized by using 32 processors of the cluster and lasts about 2 hours (it depends on the number of mesh elements).

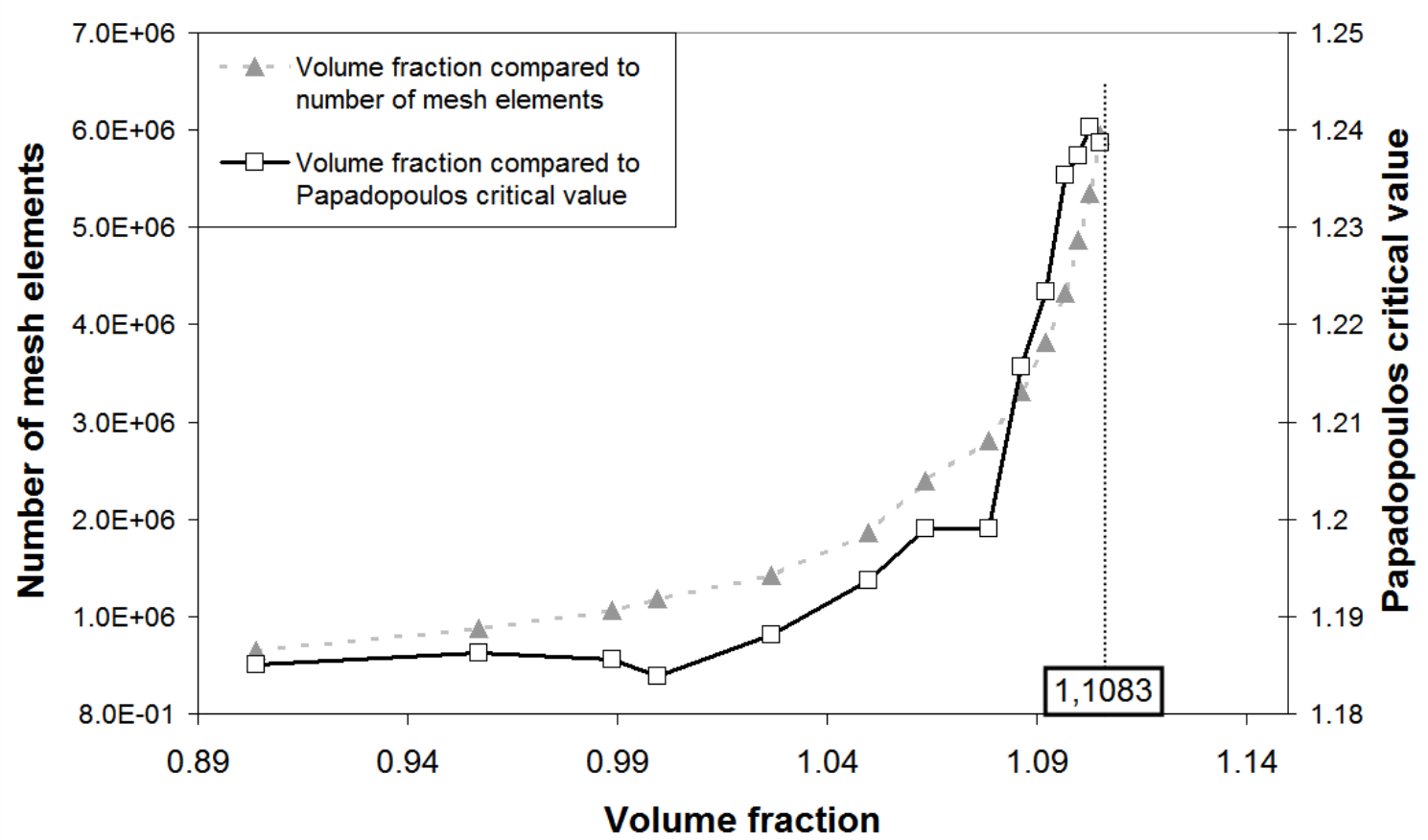

Figure 8: Evolution of the volume fraction compared to the number of mesh elements and the Papadopoulos critical value during simulations. 


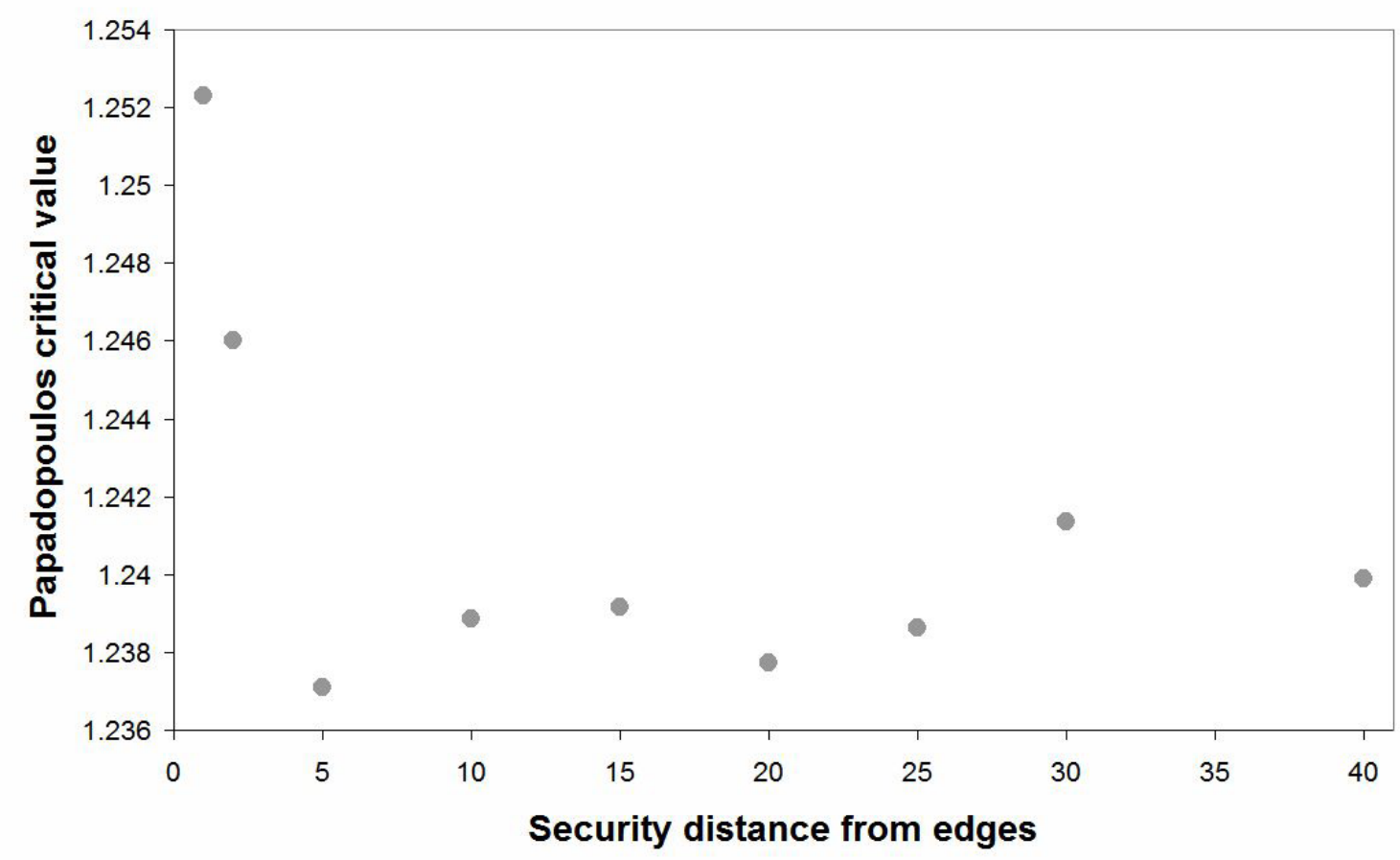

Figure 9: Influence of security distance on the prediction of the Papadopoulos criterion.

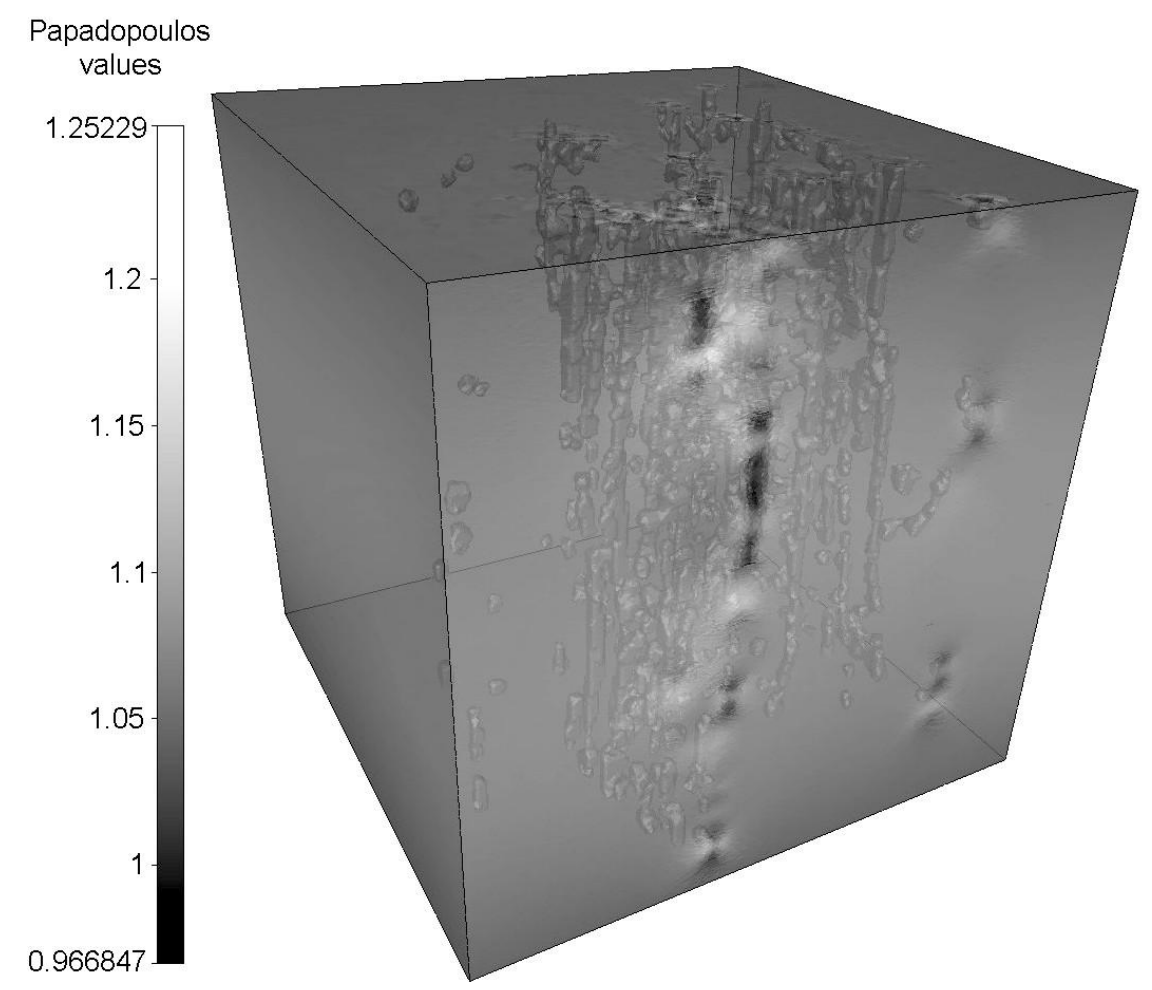

Figure 10: Stress concentrations appearing on the REV surface due to the short security distance $(1 \mu \mathrm{m})$. 
The statistical draw of Young modulus has a high impact on results can be noticed in figure 11, even if the standard deviation is relatively small. To obtain a representative volume, it is important to take into account this sensitive parameter by decreasing its effects from a statistical study. One simulation with only one set of material parameters is not sufficient to describe the statistical effects.

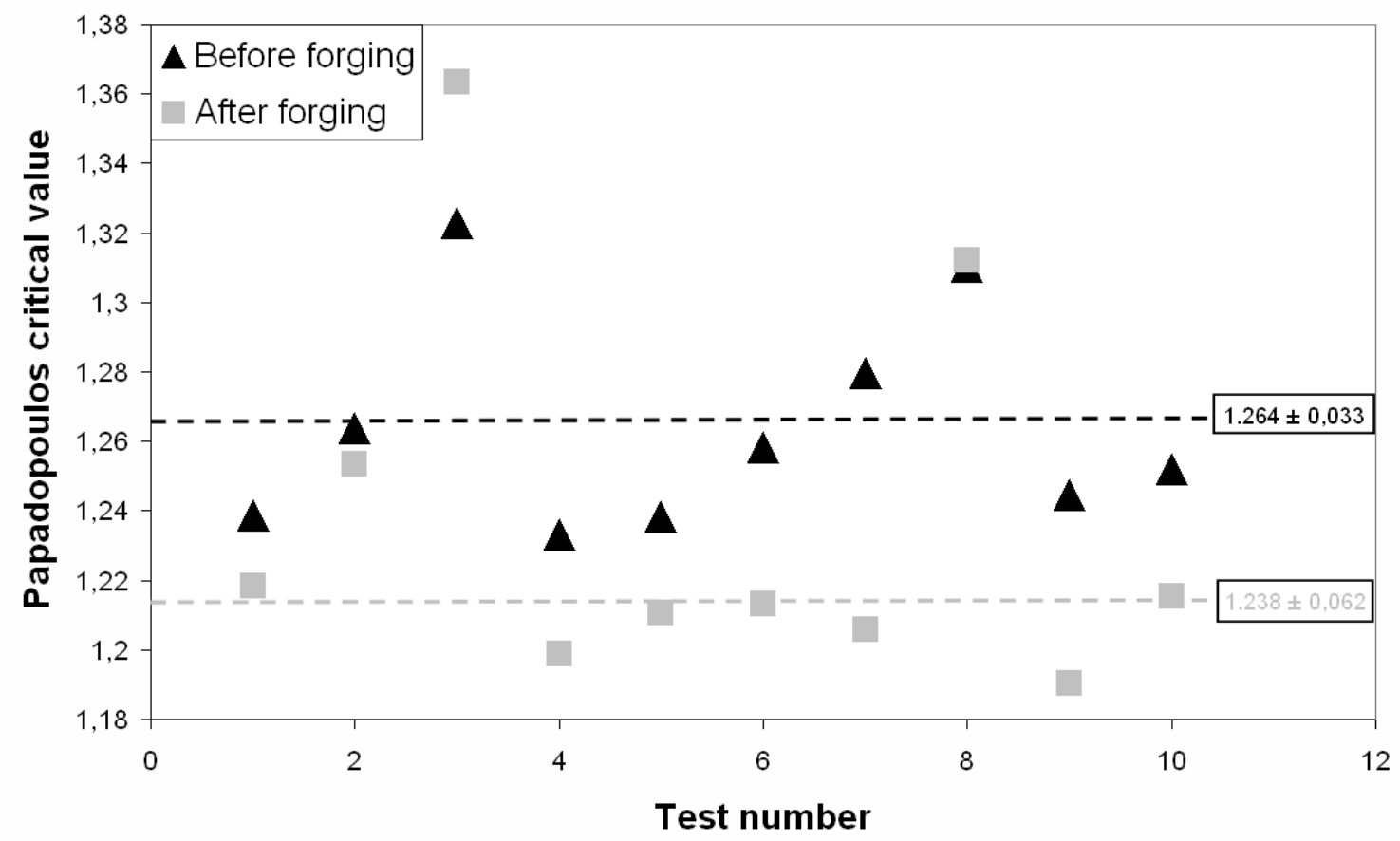

Figure 11: Papadopoulos critical value depending on statistical drawing of inclusion Young modulus following a normal distribution.

Figure 12 depicts the repartition of iso-values of the Papadopoulos criterion around inclusions. Out of concern for clarity, critical areas were represented by taking into account all values over 1.21 until maximal value. It can be noted that critical values are concentrated in the vicinity of particles. The critical area is not necessary located near one isolated inclusion but rather in an overlapping area. 


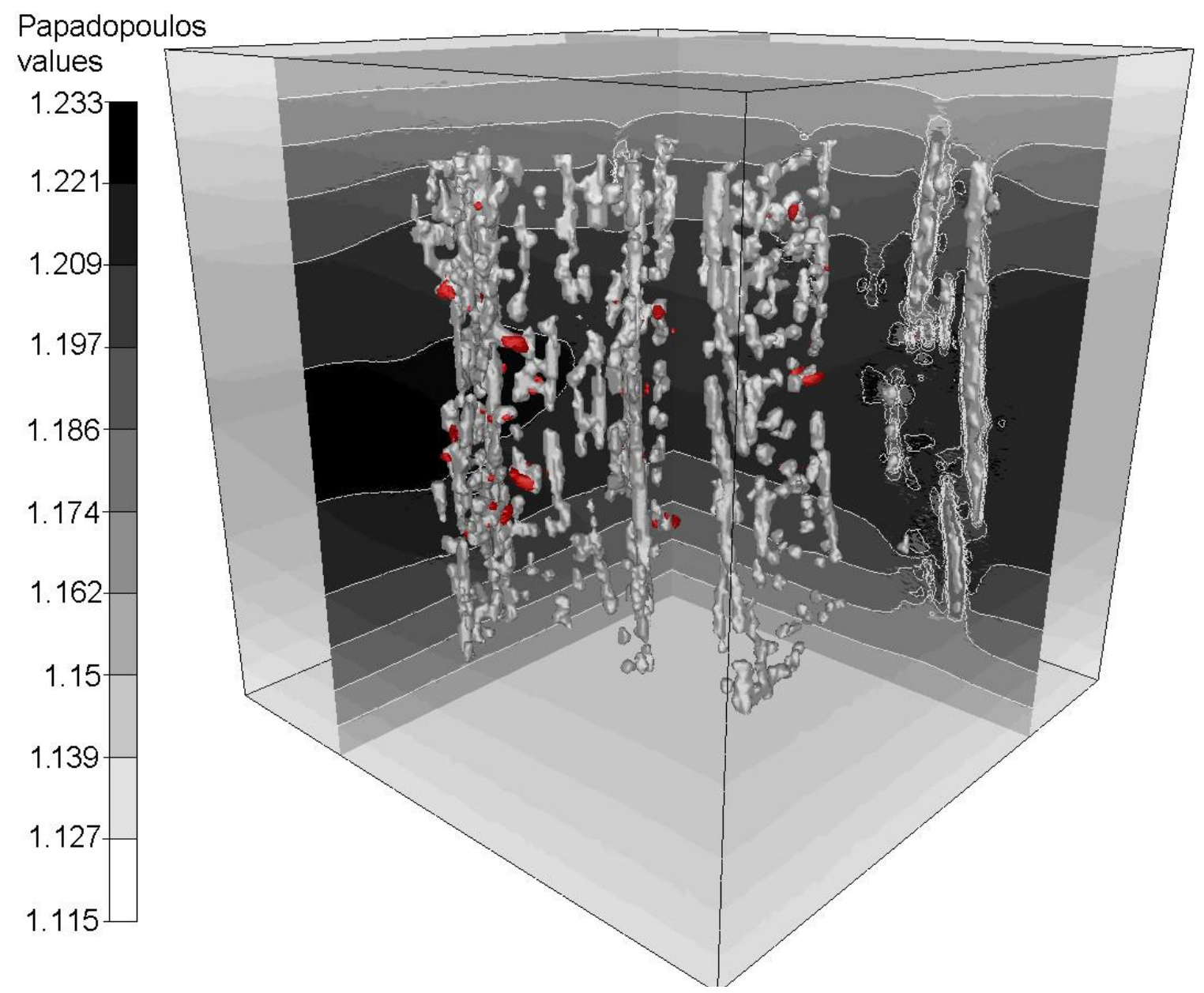

Figure 12: Example of iso-values of Papadopoulos criterion for a REV loaded with only one sinusoidal cycle. 


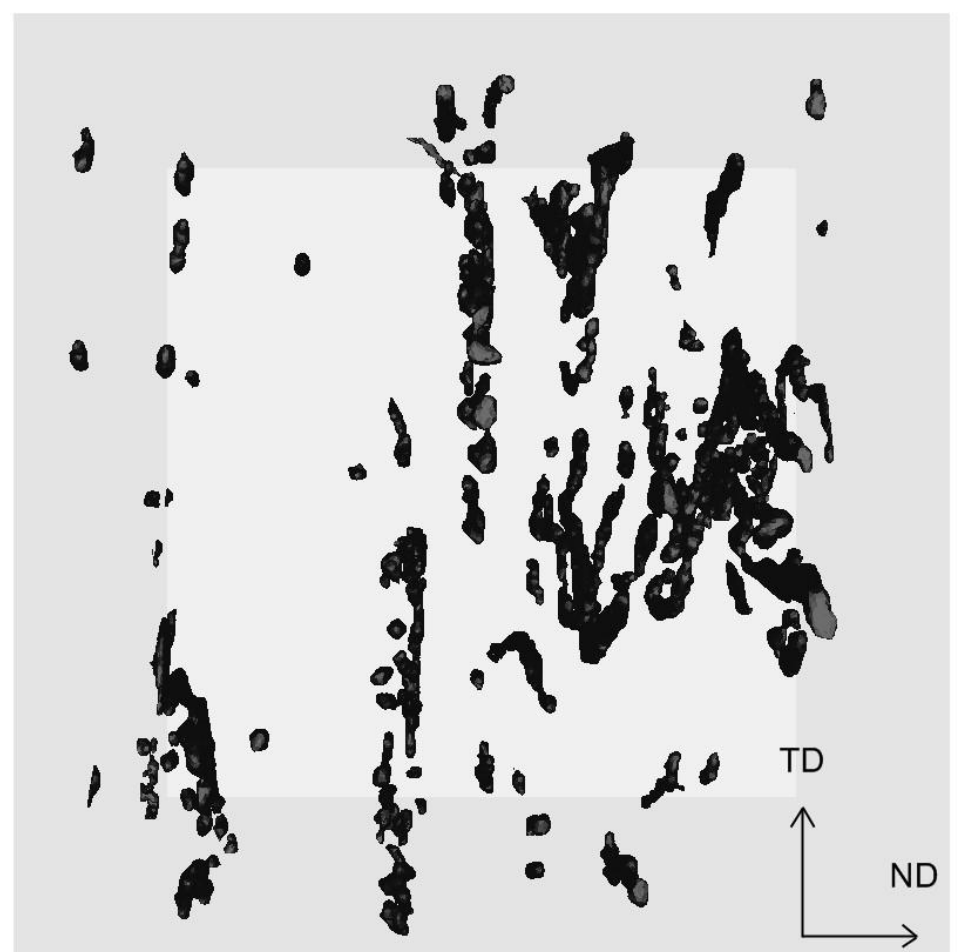

(a)

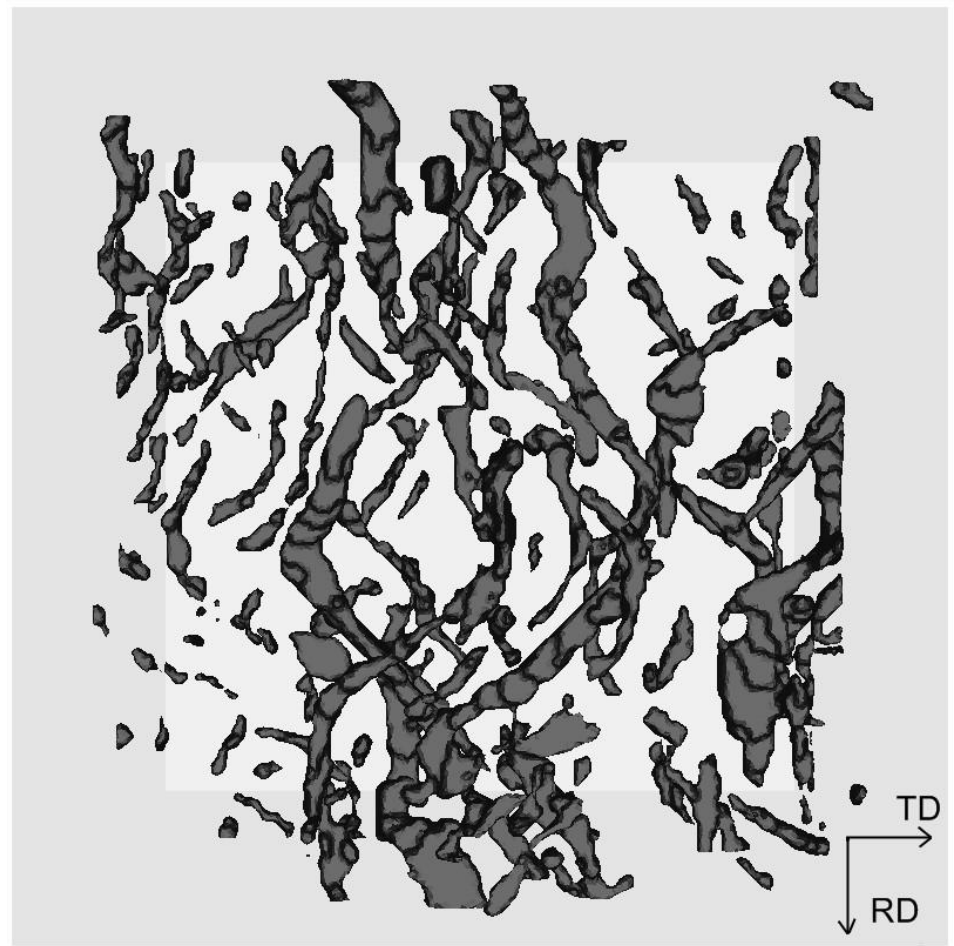

(b)

Figure 13: Example of morphological characteristics cutting from the forging part represented in a) the normal direction (ND) - transverse direction (TD) and in b) the rolling direction (RD) transverse direction (TD). 
The morphology of inclusions has also been studied by selecting a tomography probe of the forged component composed of 197 inclusions (see figure 13). In this case, the shape of inclusions is extremely deformed. The goal is then to confront the Hessian strategy approach with more complicated form than the non-forged case. It can be noticed that the tortuosity reaches a high level of distortion. As for the previous case, the first simulation with such configuration was performed on a $102 \times 102 \times 102 \mu \mathrm{m}^{3}$ REV defining a security distance of $1 \mu \mathrm{m}$. A similar analysis was performed to determine the influence of the number of mesh elements on the calculation of the Papadopoulos critical value (figure 14). The evolution has an exponential form when a certain number of mesh elements is reached, and tends to stabilize towards 2,600,000 elements. The optimized number of elements is less than in the previous case with the same remeshing parameters. If this value is compared to volume fraction given in figure 15, the evolution of the Papadopoulos critical volume and the number of mesh elements are both linked by an alike progressive way.

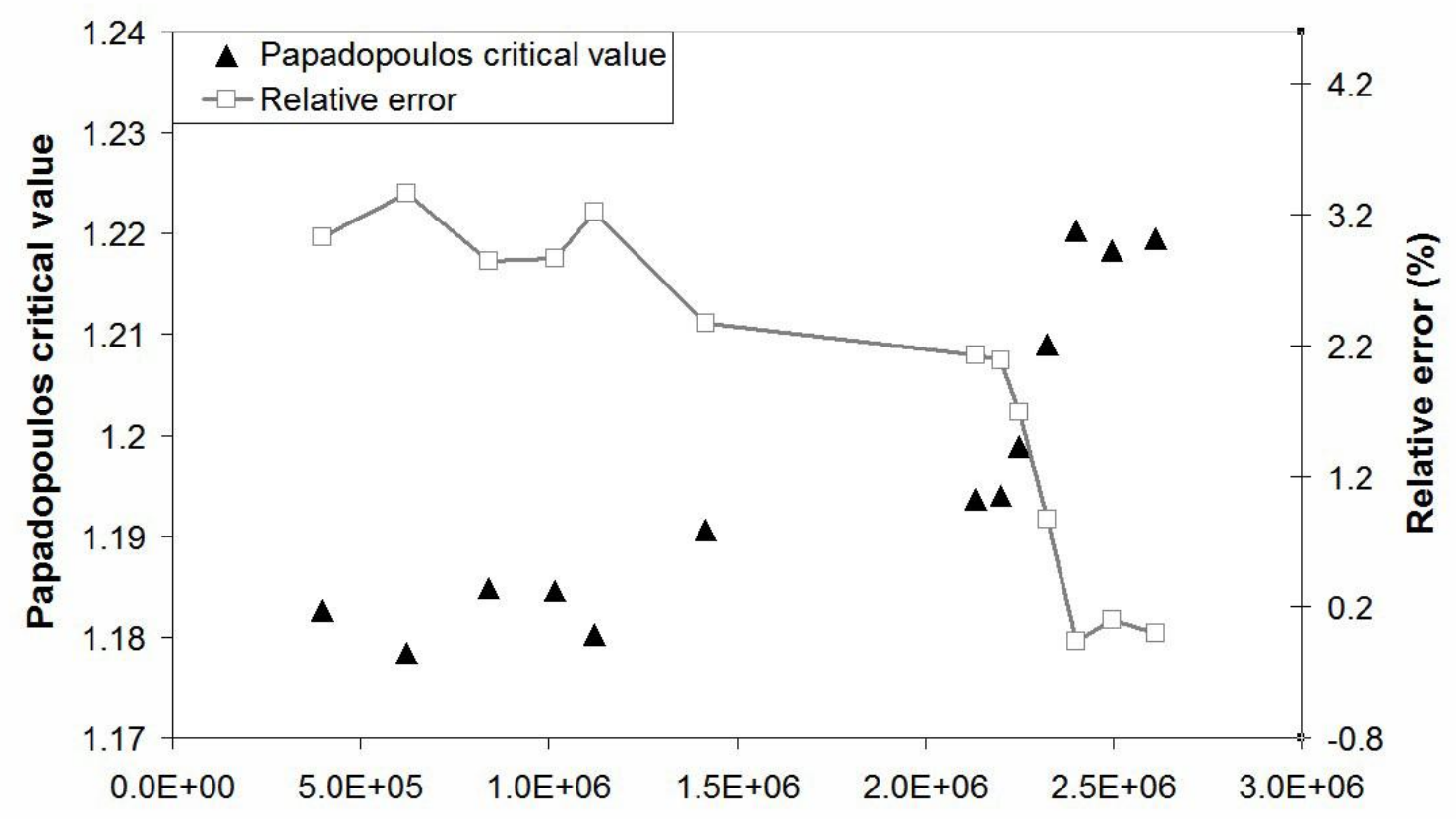

Number of mesh elements

Figure 14: Evolution of the Papadopoulos critical value depending on the remeshing method increments for the forging part. 


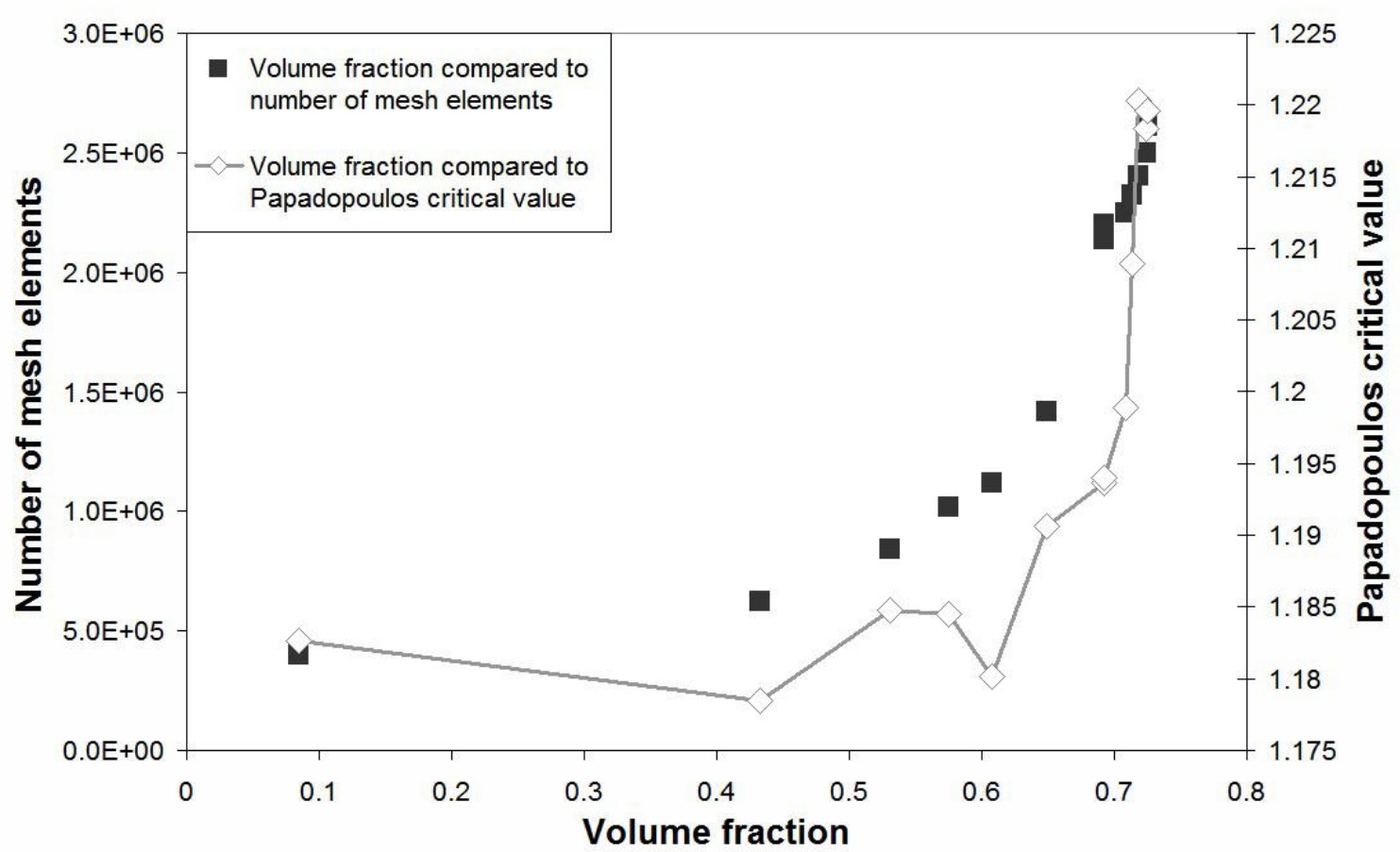

Figure 15: Evolution of the volume fraction compared to the number of mesh elements and the Papadopoulos critical value during simulations.

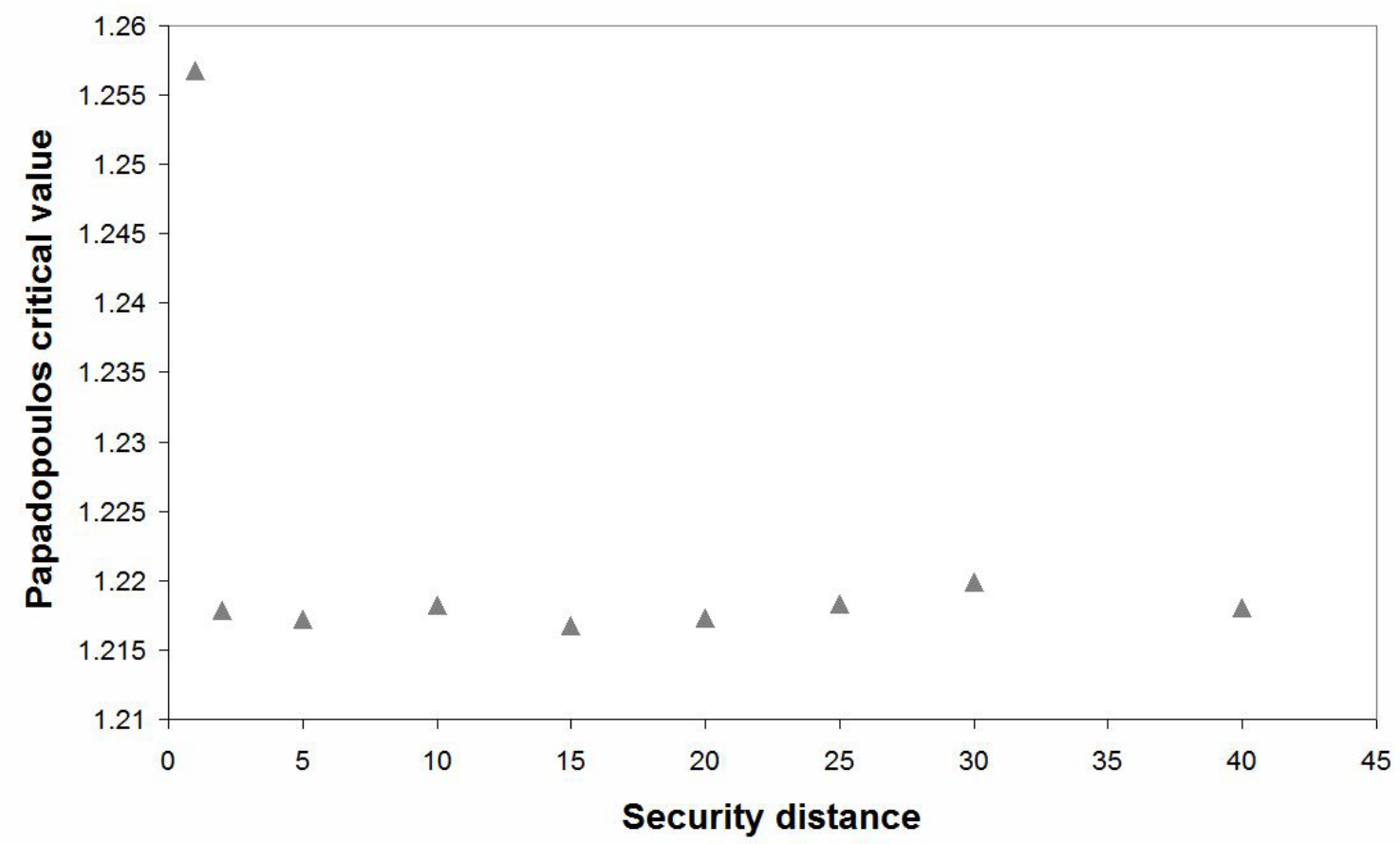

Figure 16: Influence of security distance on the prediction of the Papadopoulos criterion. 
For this kind of structure, the minimum security distance for which no "wall effects" appear is about $2 \mu \mathrm{m}$ as we can see in figure 16 . Critical values stabilize after this threshold. The critical value is determined between inclusions with high concentration of inclusions. Figure 17 depicts an example of simulation for one cyclic loading.

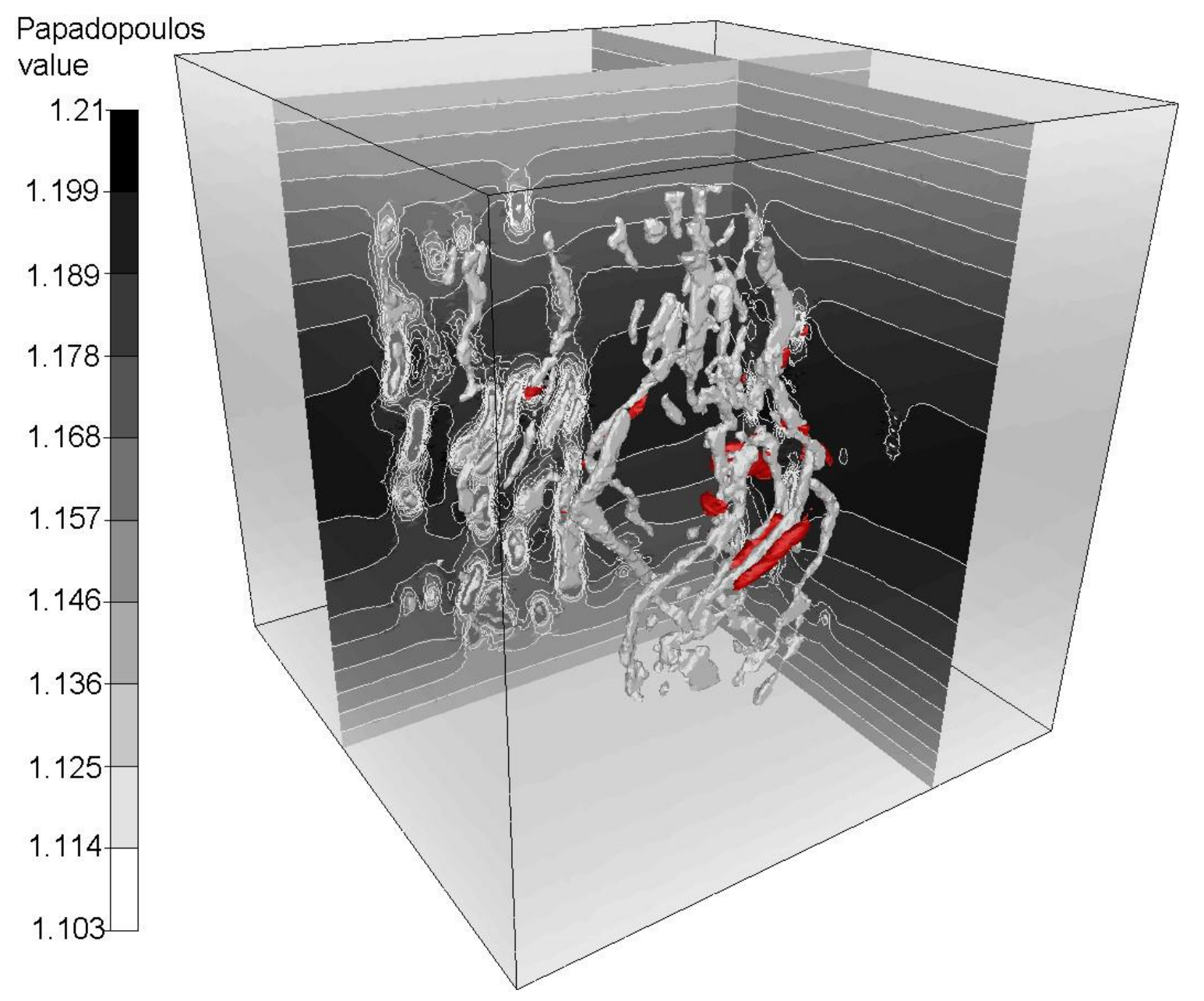

Figure 17: Critical area represented by black areas predicted by the Papadopoulos criterion for a REV loaded with only one sinusoidal cycle.

The influence of Young modulus is dramatically important as for the previous case. It can be noted in figure 11 that for a critical draw, the critical value raises up dramatically and even exceeds the maximum value determined for a geometry which seems more critical for the fatigue resistance. Material parameters are thus extremely sensitive parameters that have to be studied carefully. An optimization strategy based on a statistical study would give more reliable results.

\section{CONCLUSION AND PERSPECTIVES}


We propose in this work a former step in the validation of microstructural tomographic approaches, especially in the case of fatigue and damage calculations. An advanced anisotropic mesh adaptation method is presented and used here to reconstruct 3D complex inclusions embedded in a representative elementary volume. Starting from tomographic probe, the proposed approach allows a simple and fast immersion of the inclusions using the levelset function. The anisotropic mesh adaptation, based on a Hessian strategy is able to capture all the inclusions for any degree of complexity and irregularity in their respective shapes. The control of number of elements and parallel computing highlight the potential of the proposed approach to handle complex tomographic reconstruction.

A sensitivity analysis, based on a large number of numerical computations, has been then carried out to optimize the REV. It takes into consideration the influences of the number of elements, the inclusion volume fraction, the security distance parameter and the material parameters. The good agreement between the sensitivity analysis and the numerical results shows that the method is fairly independent from mesh and "wall-effects". Additionally, some results show that we still have an important dependency from materials inclusion properties.

Mechanical and thermal residual stresses at the interface between matrix and inclusions could be investigated in a future works. However, to perform such simulations, experimental input data have to be determined. Some of them are still extremely difficult to obtain with current experimental methods. The way to circumvent that situation is to take into account the statistical response of the material.

\section{ACKNOWLEDGEMENT}

This work has been performed within the ANR (French National Research Agency) Optiforge project, in collaboration with several industrial partners (Ascoforge, Ascometal, Cetim, PSA, Setforge, Transvalor) and academic institutions (ENSAM d'Angers, INSA Lyon, ENSMP - CEMEF).

The authors are very grateful to C. Verdu and E. Maire of INSA Lyon for their contribution in obtaining tomographic REVs. Our acknowledgements are also due to $\mathrm{H}$. Digonnet for his great contribution in numerical algorithms of mesh refinement.

\section{References}


[1] B. Mather, Shape, Surface texture and coatings, Significance of Tests and Properties of Concrete and Concrete Aggregates, ASTM STP 169, American Society of Testing Materials, 1955.

[2] I. Comby-Peyrot, Development and validation of 3D computational tool to describe damage and fracture due to alkali silica reaction in concrete structures, PhD thesis, Ecole Nationale Supérieure des Mines de Paris, 2006.

[3] H. Sadouki, F. H. Wittmann, On the analysis of the failure process in composite materials by numerical simulation, Mat. Science Engng 104 (1988) 920.

[4] P. E. Roelfstra, A numerical approach to investigate the properties of concrete: Numerical concrete, PhD thesis, Ecole Polytechnique Fédérale de Lausanne EPFL, 1989.

[5] J. Segurado, C. Gonzàlez, J. Llorca, A numerical investigation of the effect of particle clustering on the mechanical properties of composites, Acta Mater. 51 (2003) 2355-2369.

[6] J. Segurado, J. Llorca, A new three-dimensional interface finite element to simulate fracture in composites, Int. J. Solids \& Structures 41 (2004) 2977-2993.

[7] S. Häfner, S. Eckardt, T. Luther, C. Könke, Mesoscale modeling of concrete: geometry and numerics, Comput. Struct. 84 (2006) 450-461.

[8] Z. M. Wang, A. K. H. Kwan, A. C. Chan, Mesoscopic study of concrete I: generation of random aggregate structure and finite element mesh, Comput. Struct. 70 (1999) 533-544.

[9] N. Yang, J. Boselli, I. Sinclair, Simulation and quantitative assessment of homogeneous and inhomogeneous particle distributions in particulate metal matrix composites, Journal of microscopy 201 (2001) 189-200.

[10] M. Milesi, Impact des caractéristiques microstructurales des pièces forgées sur leur tenue en fatigue à grand nombre de cycles : Modélisation multi-échelles et validation expérimentale, PhD thesis, Ecole Nationale Supérieure des Mines de Paris, 2009.

[11] M. Milesi, Y. Chastel, E. Hachem, M. Bernacki, R. E. Logé, P. O. Bouchard, A multi-scale approach for high cycle anisotropic fatigue resistance: Application to forged components, Mater. Sci. Eng. A 527 (18-19) (2010) 4654-4663.

[12] N. Chawla, V. V. Ganesh, B. Wunsch, Three-dimensional (3d) microstructure visualization and finite element modeling of the mechanical behavior of $\mathrm{SiC}$ particle reinforced aluminum composites, Scripta Mater. 51 (2004) 161-165. 
[13] N. Chawla, R. S. Sidhu, V. V. Ganesh, Three-dimensional visualization and microstructure-based modeling of deformation in particle-reinforced composites, Acta Mater. 54 (2006) 1541-1548.

[14] A. Stiénon, A. Fazekas, J. Y. Buffière, A. Vincent, P. Daguier, F. Merchi, A new methodology based on $x$-ray micro-tomography to estimate stress concentrations around inclusions in high strength steels, Mater. Sci. Eng. A 513 (2009) 376-383.

[15] G. Laschet, J. Sauerhering, O. Reutter, T. Fend, J. Scheele, Effective permeability and thermal conductivity of open-cell metallic foams via homogenization on a microstructure model, Computational Materials Science 45 (2009) 597-603.

[16] I. M. Gitman, H. Askes, L. J. Sluys, Representative volume: Existence and size determination, Engng Fract. Mech. 74 (2006) 2518-2534.

[17] J. A. Sethian, Level Set Methods and Fast Marching Methods, Cambridge University Press, Cambridge, 1996.

[18] L. Ville, L. Silva, T. Coupez, Convected level set method for the numerical simulation of fluid buckling, Int. J. Numer. Meth. Fluids (2010) n/a doi:10.1002/d.2259.

[19] R. Valette, J. Bruchon, H. Digonnet, P. Laure, M. Leboeuf, L. Silva, B. Vergnes, T. Coupez, Méthodes d'interaction fluide-structure pour la simulation multi-échelles des procédés de mélange, Mec. Ind. 8 (3) (2007) 251-258.

[20] J. Bruchon, H. Digonnet, T. Coupez, Using a signed distance function for the simulation of metal forming processes: Formulation of the contact condition and mesh adaptation. from a lagrangian approach to an eulerian approach, Int. J. Numer. Methods Eng. Wiley InterScience 78 (8) (2009) 980-1008.

[21] M. Bernacki, Y. Chastel, T. Coupez, R.E. Logé, Level set framework for the numerical modelling of primary recrystallization in polycrystalline materials, Scripta Mater. 58 (12) (2008) 1129-1132.

[22] E. Hachem, T. Kloczko, H. Digonnet, T. Coupez, Stabilized finite element solution to handle complex heat and fluid flows in industrial furnaces using the immersed volume method, International Journal for Numerical Methods in Fluids (2010) n/a doi:10.1002/d.2498.

[23] M. Bernacki, H. Resk, T. Coupez, R. E. Logé, Finite element model of primary recrystallization in polycrystalline aggregates using a level set framework, Modelling Simul. Mater. Sci. Eng. 17 (6) (2009) 064006. 
[24] W. C. Oliver, G. M. Pharr, An improved technique for determining hardness and elastic modulus using load and displacement sensing indentation experiments, J. Mater. Res. 7 (1992) 1564-1583.

[25] H. Digonnet, L. Silva, T. Coupez, Cimlib: a fully parallel application for numerical simulations based on components assembly, in: NUMIFORM '07 proceedings, American Institute of Physics, J.M.A. Cesar de Sa and A.D. Santos Editors, 2007, p. 269274.

[26] J. L. Chenot, F. Bay, An overview of numerical modelling techniques, J. Mater. Proc. Tech. 80-81 (1998) 8-15.

[27] I. V. Papadopoulos, Fatigue limit of metals under multiaxial stress conditions: the microscopic approach, Technical Note N $\mathrm{o}$. 1.93.101, Commission of the European Communities, Joint Research Centre.

[28] K. D. Van, B. Griveau, O. Message, On a new multiaxial high cycle fatigue limit criterion: Theory and application, Mechanical Engineering Publications EGF 3 (1989) 479-496.

[29] F. Morel, L. Flaceliere, Data scatter in multiaxial fatigue: from the infinite to the finite fatigue life regime, Int. J. Fatigue 27 (2005) 1089-1101.

[30] G. E. P. Box, M. E. Müller, A note on the generation of random normal deviates, Annals of Mathematical Statistics 29 (1958) 610-611.

[31] M. Matsumoto, T. Nishimura, Mersenne twister: A 623-dimensionally equidistributed uniform pseudorandom number generator, ACM Transactions on Modelling and Computer Simulations 8 (1998) 3-30.

[32] M. Lemaire, Fiabilité des structures : Couplage mécano-fiabiliste statique, Hermes-Lavoisier, Paris, 2005.

[33] M. Bernacki, Y. Chastel, H. Digonnet, H. Resk, T. Coupez, R. E. Logé, Development of numerical tools for the multiscale modelling of recrystallization in metals, based on a digital material framework, Computer Methods in Materials Science 7 (2007) 142-149.

[34] M. Milesi, Y. Chastel, M. Bernacki, R. E. Logé, P. O. Bouchard, Explicit microscopic fatigue analysis of forged components, Computer Methods in materials science 7 (2007) 383-388.

[35] M. Milesi, Y. Chastel, E. Hachem, M. Bernacki, R. E. Logé, P. Bouchard, Digital microstructures matching statistical distributions of features in real materials _ example of forgings, Steel Research International 81 (9) (2010) 14421445. 
[36] L. A. Feldkamp, L. C. Davis, J. W. Kress, Practical cone-beam algorithm, J. Opt. Soc. A 1 (1984) 612-619.

[37] G. Kunert, Toward anisotropic mesh construction and error estimation in the finite element method, Numerical Methods for Partial Differential Equations 18 (2002) 1328-1355.

[38] F. Alauzet, P. J. Frey, P. L. George, Anisotropic mesh adaptation for rayleigh-taylor instabilities, in: European Congress on Computational Methods in Applied Sciences and Engineering (ECCOMAS), 2004.

[39] M. Hamide, E. Massoni, M. Bellet, Adaptive mesh technique for thermalmetallurgical numerical simulation of arc welding processes, Int. J. Numer. Methods Eng. 73 (2008) 624-641.

[40] M. Fortin, Estimation d'erreur à posteriori et adaptation de maillages, Revue européenne des éléments finis 9(4) (2000) 467-486.

[41] C. Gruau, T. Coupez, 3d tetrahedral, unstructured and anisotropic mesh generation with adaptation to natural and multidomain metric, Comput. Meth. Appl. Mech. Eng. 194 (2005) 4951-4976. 